\title{
Timothy Williamson über analytische Wahrheiten und den Gegenstandsbereich der Philosophie
}

\section{$1 \quad$ Einleitung}

Timothy Williamson argumentiert in The Philosophy of Philosophy gegen eine Position, die er »Philosophischer Exzeptionalismus« nennt: die Auffassung, dass sich die Philosophie entweder in Bezug auf ihren Gegenstandsbereich (subject matter) oder in Bezug auf ihre Methode beziehungsweise die Art der Evidenz, auf die sie sich abstützt, grundlegend von anderen Wissenschaften unterscheidet. Entgegen einem verbreiteten Irrglauben, so Williamsons Auffassung, handeln philosophische Wahrheiten nicht nur oder hauptsächlich von Begriffen. ${ }^{1}$ Außerdem ist die Philosophie Williamson zufolge im Gegensatz zu den Naturwissenschaften zwar im Wesentlichen ein Lehnstuhl-Unterfangen. Aber damit unterscheidet sie sich nur graduell von den Naturwissenschaften. Und die Evidenz, auf die sich Philosophinnen und Philosophen berufen, und ihre Vorgehensweise sind nicht kategorial verschieden von der Evidenz und der Vorgehensweise von Naturwissenschaftlern (und der Evidenz und der Vorgehensweise, mittels derer wir in alltäglichen Erwägungen außerhalb der Wissenschaften Erkenntnisse erlangen).

Für die vorliegende Abhandlung sind zwei Themen aus Williamsons Buch besonders interessant: Erstens die Behauptung, dass philosophische Fragen nicht nur nicht explizit, sondern auch nicht implizit von sprachlichen Ausdrücken oder von Begriffen handeln. Zweitens das Thema Analytizität, mit dem sich Williamson über fast hundert Seiten hinweg in zwei Kapiteln auseinandersetzt. Williamson argumentiert in diesen Kapiteln nicht grundsätzlich gegen die Existenz analytischer Wahrheiten. Er meint, dass es unproblematische Konzeptionen von Analytizität gibt, insbesondere die so genannte »Frege-Analytizität«, unter Rückgriff auf die sich beispielsweise der Satz »Junggesellen sind unverheiratete Männer« als analytisch herausstellen lässt. Williamson argumentiert aber sowohl gegen die Auffassung, dass es

1 Interessanterweise legt Williamson nahe, dass philosophische Wahrheiten von allen Arten von Gegenständen in der Welt handeln können. Wäre dies der Fall, ließe sich mit Bezug auf diesen Umstand allerdings sicher ein philosophischer Exzeptionalismus begründen. Denn keine andere Wissenschaft hat einen derart umfassenden Gegenstandsbereich. 
metaphysisch analytische Sätze gibt, die allein aufgrund ihrer Bedeutung wahr sind, als auch gegen die Auffassung, dass es epistemologisch analytische Sätze oder Gedanken gibt - Sätze also, die man nicht verstehen, oder Gedanken, die man nicht fassen kann, ohne sie für wahr zu halten.

Das Thema Analytizität bespricht Williamson immer unter dem Blickwinkel eines bestimmten Versuchs, einen philosophischen Exzeptionalismus zu begründen: Er argumentiert gegen eine Position, der zufolge sich ein methodologischer Exzeptionalismus der Philosophie unter Rückgriff darauf begründen lässt, dass ein zentraler Teil der Wahrheiten, auf die sich die Philosophie beruft - anders als in den anderen Wissenschaften - analytisch ist. Williamsons Argument gegen diese Idee funktioniert, grob gesagt, wie folgt: Entweder diese Kernwahrheiten der Philosophie sind analytisch im oben erwähnten, unproblematischen Sinn. Dann können wir mit Bezug auf sie nicht unmittelbar einen philosophischen Exzeptionalismus begründen, weil der Umstand, dass ein Urteil beispielsweise Frege-analytisch ist, für sich allein nichts darüber aussagt, auf welche Weise wir die Wahrheit des Urteils erkennen können. Oder die Kernwahrheiten der Philosophie sollen epistemologisch analytisch sein. Diese These hat zwar (wenigstens prima facie) Implikationen in Bezug auf die Art und Weise, in der wir entsprechendes Wissen erlangen, und ist damit der aussichtsreichste Kandidat für eine Begründung eines philosophischen Exzeptionalismus. Aber wie sich zeigt, so Williamson, ist diese These nicht haltbar, da es keine epistemologisch analytischen Urteile gibt.

Das vorliegende Kapitel ist wie folgt aufgebaut: Im Abschnitt 2 rekonstruiere und kritisiere ich nach einem kurzen Blick auf die Geschichte der Frage nach dem Gegenstandsbereich der Philosophie Williamsons Argumente dafür, dass philosophische Probleme typischerweise nicht von sprachlichen Ausdrücken oder Begriffen handeln. Außerdem setze ich mich mit der Frage auseinander, was überhaupt damit gemeint sein kann, dass philosophische Probleme von Wörtern, von Begriffen oder von Gegenständen in der Welt handeln. Da die Bedeutung solcher Formulierungen nicht ganz klar ist, könnte man auf die Idee kommen, dass der diesbezügliche Konflikt zwischen Williamson und den Vertreterinnen und Vertretern einer begriffsanalytischen Philosophie-Konzeption nur ein Streit um Worte ist. Ich argumentiere allerdings gegen diese Idee und versuche zu zeigen, dass zwischen den beiden Parteien in der Tat eine substanzielle Meinungsverschiedenheit in Bezug auf den Gegenstandsbereich der Philosophie besteht.

Im Abschnitt 3 befasse ich mich mit Williamsons Diskussion von metaphysischen oder semantischen Auffassungen von Analytizität. Besonders ausführlich gehe ich dabei auf die Konzeption ein, nach welcher analytische Wahrheiten wahr aufgrund ihrer Bedeutung sind: Ergibt die Formulierung 
»wahr aufgrund von Bedeutung « contra Williamson Sinn? Und trifft es zu, dass analytische Wahrheiten allein aufgrund ihrer Bedeutung wahr sind, währenddem synthetische Wahrheiten wahr sind einerseits aufgrund ihrer Bedeutung und andererseits aufgrund dessen, was (in der Welt) der Fall ist?

Der Abschnitt 4 ist Williamsons Argumenten gegen die Idee gewidmet, dass sich analytische Wahrheiten adäquaterweise charakterisieren lassen als solche Wahrheiten, bei denen es nicht möglich ist, sie zu verstehen, ohne ihnen zuzustimmen. Im Zentrum von Williamsons Diskussion einer solchen epistemologischen Konzeption von Analytizität steht sein Beispiel zweier Philosophen, Peter und Stephen, die angeblich beide den Satz »Alle Füchsinnen sind Füchsinnen« verstehen, ohne ihm zuzustimmen. Ich versuche, zu zeigen, dass Williamsons Argumentation in diesem Zusammenhang nicht stichhaltig ist. Im Zusammenhang mit der Frage, was es überhaupt bedeuten würde, zu glauben, dass nicht alle Füchsinnen Füchsinnen sind, setze ich mich außerdem mit einem Artikel von Tyler Burge auseinander, auf den sich Williamson im Kontext seiner diesbezüglichen Argumentation beruft.

\section{Der Gegenstandsbereich der Philosophie}

\section{Die linguistische Philosophie und die Frage nach ihrem Gegenstandsbereich}

Sind Vertreterinnen und Vertreter einer Philosophie-Konzeption der Art, wie sie im vorliegenden Buch verteidigt wird, - oder auch Vertreterinnen und Vertreter einer Ideal Language-Philosophie - auf die Auffassung festgelegt, dass philosophische Probleme von sprachlichen Ausdrücken handeln? Um diese Frage sowie die damit zusammenhängende Frage, ob eine Analyse von Bedeutungen oder Begriffen uns irgendwelche Aufschlüsse über die außersprachliche Welt liefern kann, dreht sich schon seit der Entstehung dieser Strömungen eine brisante Debatte. In Richard Rortys The Linguistic Turn (1967), der wohl wichtigsten Sammlung von Artikeln für und gegen verschiedene Ausprägungen der so genannten »Linguistischen Philosophie«, berühren fast alle Texte diese Fragen in kleinerem oder größerem Ausmaß, und der titelgebende Kritikpunkt von Ernest Gellners berüchtigtem Pamphlet Words and Things (1959) besteht ebenfalls darin, dass Vertreterinnen und Vertreter der Ordinary Language-Philosophie ihre Zeit damit verschwenden, sich mit Wörtern zu befassen, statt sich den Dingen zuzuwenden, auf die sich die Wörter beziehen.

In The Linguistic Turn formulieren aber auch bereits mehrere Philosophen Zweifel an der Einteilung von Fragen oder Problemen in solche, die von Wörtern handeln, und solche, die von der Welt handeln. So argumentiert zum 
Beispiel Stuart Hampshire in »Are All Philosophical Questions Questions of Language? « dafür, dass es viele Fragen gibt, die sowohl sprachliche Ausdrücke als auch Tatsachen betreffen. Wenn wir beispielsweise fragen: »Ist diese Blume eher mauvefarben oder violett?«, dann stellen wir gleichermaßen eine empirische Frage über diese Blume wie eine Frage nach der korrekten Ver-

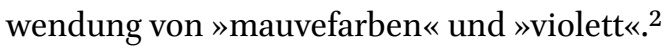

Roderick Chisholm macht in seinem Beitrag zu Rortys Sammelband geltend, dass nicht klar sei, was überhaupt unter einer empirischen Aussage zu verstehen sei. Er prüft verschiedene mögliche Interpretationen von »empirisch« und verteidigt die These, dass es naheliegende Lesarten von »empirisch « gibt, nach welchen diverse synthetische Aussagen nicht empirisch sind, und umgekehrt auch naheliegende Lesarten von »empirisch «, nach welchen bestimmte begriffliche Wahrheiten als empirisch zu klassifizieren wären. $\mathrm{Ob}$ philosophische Aussagen, Fragen oder Probleme empirisch seien oder nicht, ist angesichts dieses Interpretationsspielraums eine erläuterungsbedürftige Frage. $^{3}$

In mehreren Artikeln aus The Linguistic Turn wird darauf hingewiesen, dass philosophische Aussagen in der Regel jedenfalls den Anschein von Urteilen über die Welt haben. ${ }^{4}$ Wenn wir sagen, dass eine Person nicht an zwei Orten gleichzeitig sein kann, schreibt beispielsweise John Wisdom, haben wir nicht den Eindruck, über Wörter zu sprechen. ${ }^{5}$ Wenn wir darauf bestehen, dass die Aussage »Eine Person kann nicht an zwei Orten gleichzeitig sein« eigentlich vom Wort »Person « (in Kombination mit den anderen involvierten Wörtern) handelt oder jedenfalls wahr ist aufgrund der Art und Weise, wie dieses Wort (und andere Wörter) verwendet wird, dann sind wir mit einem Problem konfrontiert, das Richard Hare in »Philosophical Discoveries« (1960), ebenfalls wiederabgedruckt im Rorty-Band, diskutiert: Würde daraus, dass philosophische Aussagen aufgrund von Konventionen beziehungsweise aufgrund der Art und Weise, wie wir sprachliche Ausdrücke verwenden, wahr oder falsch sind, nicht folgen, dass philosophische Aussagen kontingent sind? Denn schließlich ist es nicht notwendig, dass wir die Konventionen haben, die wir haben, respektive dass wir unsere Ausdrücke so verwenden, wie wir sie verwenden. ${ }^{6}$ Hares Replik, übertragen auf das obige Beispiel, lautet wie folgt: »Eine Person kann nicht an zwei Orten gleichzeitig sein« ist insofern

2 Vgl. Hampshire 1948, S. 285 f.

3 Vgl. Chisholm 1951, S. $178 f$.

4 Vgl. z. B. Wisdom 1937, S. 102, 105, Ambrose 1952, S. 147.

5 Vgl. Wisdom 1937, S. 101 .

6 Vgl. Hare 196o, S. $206 f$. 
notwendig, als etwas, das an zwei Orten gleichzeitig sein kann, nicht korrekterweise eine Person genannt werden kann. ${ }^{7}$

Vor dem Hintergrund der hier verteidigten Philosophie-Konzeption könnte man die Notwendigkeit des zur Diskussion stehenden Satzes etwas ausführlicher wie folgt begründen: Wenn wir die Art und Weise, wie wir sprachliche Ausdrücke verwenden, voraussetzen (was wir normalerweise machen, wenn wir die Wahrheit oder Falschheit einer Aussage beurteilen ${ }^{8}$ ), dann kann »Eine Person kann nicht an zwei Orten gleichzeitig sein « nicht falsch sein. Selbstverständlich könnten wir unsere sprachlichen Konventionen derart verändern, dass die Wortfolge »Eine Person kann nicht an zwei Orten gleichzeitig sein« hinterher etwas Falsches oder Kontingentes zum Ausdruck bringen würde. Aber dies würde mit einer Veränderung der Bedeutung dieses Satzes einhergehen. Solange der Satz das bedeutet, was er bedeutet, ist er wahr - ebenso wie jeder Ausdruck einer anderen Sprache, der dasselbe bedeutet wie »Eine Person kann nicht an zwei Orten gleichzeitig sein « ${ }^{9}$

Genau vierzigJahre nach dem Erscheinen von The Linguistic Turn kommt die Frage nach dem Gegenstandsbereich der Philosophie bei Timothy Williamson zur Sprache, der sich ihr aus einer ganz anderen Perspektive zuwendet als die oben erwähnten Autoren. Seiner Argumentation will ich mich nun zuwenden.

\subsection{Williamsons Beispiel}

Nehmen wir an, auf dem Mars gab es früher sehr viel Wasser, im Laufe der Zeit bis heute sei die Flüssigkeit aber gleichmäßig bis auf das letzte Wassermolekül verdunstet. Wir können dann die Frage stellen:

(1) War der Mars zu jedem Zeitpunkt entweder trocken oder nicht trocken? Anhand dieses Beispiels befasst sich Williamson mit der Frage, ob philosophische Probleme im Wesentlichen von Sprache oder von Begriffen handeln. Ein Vagheits-Problem wie dasjenige betreffend die Trockenheit des Mars ist Williamson zufolge einer der aussichtsreichsten Kandidaten für ein philosophisches Problem, das implizit von Sprache oder von Begriffen handelt. Wenn selbst hier gezeigt werden kann, dass das Problem nicht von Sprache oder von Begriffen handelt, so Williamson, dann wird dies erst recht für die meisten anderen philosophischen Probleme gelten. ${ }^{10}$

$7 \quad$ Vgl. Hare 1960, S. 211.

8 Zumindest dann, wenn die Aussage nicht davon handelt, wie wir einen bestimmten sprachlichen Ausdruck verwenden.

9 Vgl. zum letzten Punkt auch Hanfling 2000, S. 65-71.

10 Vgl. Williamson 2007, S. 23 f. 
Die Frage (1) handelt, so Williamson, explizit weder von den Wörtern »trocken« oder »Mars« noch von den Begriffen trocken oder Mars, sondern vom Planeten Mars, und vielleicht noch von der Eigenschaft, trocken zu sein." Man könnte, so Williamson weiter, auf die Idee kommen, dass die Frage implizit vom Wort »trocken « handle, da sie äquivalent ist mit der folgenden Frage:

(2) Gehörte der Mars zu jedem Zeitpunkt entweder zur Extension oder zur Anti-Extension von »trocken «?

Gegen diese Argumentation formuliert Williamson drei Einwände: Erstens, auf diese Weise könnte man auch dafür argumentieren, dass eine gewöhnliche empirische Frage wie »Ist dieser Stein schwerer als ein Kilogramm?« in Wirklichkeit von sprachlichen Ausdrücken handle. Denn diese Frage ist äquivalent zu: »Gehört dieser Stein zur Extension von >schwerer als ein Kilogramm $<$ ? Zweitens, Äquivalenz ist eine symmetrische Relation. Wir könnten mit Hinweis auf die Äquivalenz von (1) und (2) also ebenso gut geltend machen, dass (2) implizit von etwas anderem als dem Ausdruck »trocken« handle, nämlich von der Eigenschaft, trocken zu sein. Drittens, wir können die Frage (1) beispielsweise auch auf Serbisch stellen, und dann ist es offensichtlich, dass sie nicht vom Wort »trocken« handelt, da dieses Wort in der serbischen Frage überhaupt nicht vorkommt. ${ }^{12}$

Analog zu (2), so Williamson, können wir auch versuchen, eine zu (1) äquivalente Frage zu formulieren, die von Begriffen statt von sprachlichen Ausdrücken handelt:

(3) Gehörte der Mars zu jedem Zeitpunkt entweder zur Extension oder zur Anti-Extension des Begriffs trocken?

Aber auch der Versuch, unter Rückgriff auf (3) geltend zu machen, dass (1) implizit von Begriffen handelt, scheitert Williamson zufolge. Die ersten zwei im vorangehenden Absatz angeführten Argumente sind nämlich auf Begriffe übertragbar: Wir können auch eindeutig empirische Fragen nach dem Muster von (3) umformulieren, und es gibt keinen Grund dafür, (3) gegenüber (1) zu privilegieren. Deshalb könnten wir ebenso gut sagen, (3) handle implizit von Trockenheit. ${ }^{13}$

Bis zu diesem Punkt ist Williamsons Argumentation nicht besonders interessant, da die Äquivalenz von (1) zu (2) und (3) offensichtlich kein aussichtsreiches Argument dafür ist, dass (1) implizit von sprachlichen Ausdrücken oder Begriffen handelt. Williamsons drittes Argument gegen die Idee, dass die Frage (1) implizit vom Wort »trocken« handelt, ist im Übrigen nicht

\footnotetext{
11 Vgl. Williamson 2007, S. 26.

12 Vgl. Williamson 2007, S. 26f., 28.

13 Vgl. Williamson 2007, S. 29 f.
} 
unproblematisch. Natürlich könnte jemand, der die Auffassung vertritt, dass (1) auf die Frage hinausläuft, ob das Wort »trocken« (korrekterweise) so verwendet wird, dass jeder Gegenstand zu jedem Zeitpunkt entweder zur Extension von »trocken « oder zur Anti-Extension dieses Wortes gehört, darauf bestehen, dass (1) nicht exakt synonym ist zur serbischen Frage:

(4) Da li je Mars uvek bio suv ili nije bio suv?

Und zwar eben deshalb, weil (4) im Gegensatz zu (1) vom Wort »suv« statt vom Wort »trocken« handelt. Severin Schroeder argumentiert zum Beispiel auf diese Weise im Zusammenhang mit den Sätzen (5) und (6), die ihm zufolge nicht genau dieselbe Bedeutung haben, da (5) vom deutschen Wort »rot«, (6) hingegen vom englischen Wort »red « handelt:

(5) Rot ist eine Farbe.

(6) Red is a colour. ${ }^{14}$

\subsection{Die Relevanz sprachlich-begrifflicher Erwägungen}

Jemand, der die Position verteidigen möchte, dass der Satz (1) implizit vom sprachlichen Ausdruck »trocken« oder vom Begriff trocken handelt, sollte sich in seiner Argumentation meines Erachtens, wie bereits erwähnt, nicht auf die Äquivalenz von (1) mit (2) respektive (3) berufen. Stattdessen sollte er oder sie geltend machen, dass wir, um die Frage zu beantworten, ob der Mars unter den eingangs geschilderten Umständen zu jedem Zeitpunkt entweder trocken oder nicht trocken war, nicht den Mars untersuchen, sondern uns Gedanken machen müssen über das Funktionieren des Ausdrucks »trocken« respektive des Begriffs trocken oder allgemein über das Funktionieren vager Prädikate und Begriffe. Dies räumt auch Williamson ein. Er schreibt bereits ganz zu Beginn des betreffenden Kapitels, dass uns eine genaue Bestimmung davon, wie viele Wassermoleküle (oder andere Flüssigkeitsmoleküle) es zu welchen Zeitpunkten auf dem Mars gab, keinen Aufschluss darüber geben würde, ob es irgendeinen Zeitpunkt gab, zu dem der Mars weder trocken noch nicht trocken war. ${ }^{15}$ Und er räumt auch ein, dass wir zur Beantwortung von (1) über Sprache und Begriffe nachdenken müssen. ${ }^{16}$ Aber er meint, dies zeige nicht, dass die Frage (1) von Sprachen oder Begriffen handle. Schließlich sei es für Fragen, die von der außersprachlichen Welt handeln, nicht untypisch, dass wir zu ihrer Beantwortung über Sprache reflektieren müssten. Wenn wir uns in einem Gerichtsfall damit auseinandersetzen, ob Smith Jones umgebracht hat, so Williamson, müssen wir uns zum Beispiel mit Zeugenaussagen befassen

\footnotetext{
14 Vgl. Schroeder 2009, S. 97-99.

15 Vgl. Williamson 2007, S. 23 f.

16 Vgl. Williamson 2007, S. 4of.
} 
und damit, ob sie eindeutig genug sind, wie aussagekräftig sie sind usw. Und Zeugenaussagen sind sprachliche Äußerungen. Ein zweites Beispiel von Williamson sind Historikerinnen und Historiker, die bei ihrer Erkundung (außersprachlicher) geschichtlicher Ereignisse sprachliche Dokumente konsultieren und sich darüber Gedanken machen müssen, welche Schlussfolgerungen aus der Existenz und dem Inhalt dieser Dokumente zuverlässig gezogen werden können. ${ }^{17}$

Diese Beispiele sind aber natürlich ganz anders geartet als unser Trockenheitsfall: Während Zeugenaussagen und die Existenz sprachlicher Dokumente aus einer bestimmten Zeit bloß Symptome beziehungsweise Indizien für das sind, was ein Gericht respektive eine Historikerin herausfinden will, ist die Weise, wie wir das Wort »trocken« verwenden, ein Kriterium dafür, welches unter den beschriebenen Umständen die korrekte Antwort auf die Frage (1) ist. ${ }^{18}$ Und dass die Beantwortung einer Frage, die vom Wort »trocken« bzw. dem Begriff der Trockenheit kriterial mit der Beantwortung der Frage (1) verbunden ist, scheint ein sehr gutes Argument dafür zu sein, dass die Frage (1) selbst von sprachlichen Ausdrücken oder Begriffen handelt. Dass die Fälle unterschiedlich geartet sind, kann außerdem wie folgt ersichtlich gemacht werden: Während wir im Gerichts- und im Historiker-Fall von Anfang an wissen, was hätte passiert sein müssen, damit es stimmt, dass Smith Jones umgebracht hat respektive dass zu dem-und-dem vergangenen Zeitpunkt dasund-das Ereignis stattgefunden hat, ist uns im Mars-Fall vor der Analyse der Art und Weise, wie das Wort »trocken« oder vage Ausdrücke im Allgemeinen verwendet werden, noch nicht klar, wie es sich um den Mars verhalten (haben) müsste, damit die Frage (1) zu bejahen ist.

Williamson scheint es in seinem Vergleich des Mars-Falls mit dem Gerichtsund dem Historiker-Fall allerdings nicht bloß darum zu gehen, dass bei der Beantwortung aller drei Sorten von Fragen auf bestimmte sprachliche Ausdrücke rekurriert werden muss. Wichtig scheint für ihn auch zu sein, dass wir in allen drei Fällen sprachliche Erwägungen anstellen müssen, die auf einer Meta-Ebene angesiedelt sind: Wenn wir uns fragen, ob der Mars zu jedem Zeitpunkt entweder trocken oder nicht trocken war, können wir, so Williamson, nicht auf der logischen Ebene bleiben und beispielsweise einfach die Regeln der klassischen Logik anwenden. Dass (1) nach diesen Regeln zu bejahen wäre, ist offensichtlich, da eine Verneinung der Frage einen logischen Widerspruch implizieren würde:

17 Vgl. Williamson 41 .

18 Für die Unterscheidung zwischen Symptom und Kriterium vgl. Glock 1996a, Eintrag »criteria«. 
(7) Es gab mindestens einen Zeitpunkt, zu dem der Mars sowohl nicht trocken als auch nicht nicht trocken war.

Wir müssen uns stattdessen auf eine metalogische Ebene begeben, auf der wir über die betreffenden Regeln der klassischen Logik nachdenken und uns fragen, ob wir an ihnen festhalten sollten oder ob es angemessen wäre, stattdessen den Regeln einer intuitionistischen, drei-wertigen oder unscharfen ( fuzzy) Logik zu folgen, nach welchen (7) entweder nicht widersprüchlich ist oder nicht von einer Verneinung der Frage (1) impliziert wird. ${ }^{19}$

So ähnlich verhält es sich nach Williamson auch bei den Fragen, die wir im Gerichts- und im Historiker-Fall beantworten wollen: Im Gerichts-Fall können wir aus dem Umstand, dass ein Zeuge behauptet, er habe beobachtet, wie Smith Jones umgebracht habe, nur Rückschlüsse darauf ziehen, ob Smith Jones umgebracht hat, nachdem wir uns auf einer Meta-Ebene darüber Gedanken gemacht haben, unter welchen Bedingungen und in welchem Maß uns Zeugenaussagen darüber Aufschluss geben können, was sich tatsächlich ereignet hat, oder, konkreter, unter welchen Umständen eine Zeugenaussage vernünftige Zweifel in Bezug darauf ausschließen kann, dass Smith Jones umgebracht hat. Analog dazu ziehen Historikerinnen und Historiker aus historischen Dokumenten nicht einfach unreflektiert Schlussfolgerungen darüber, was sich in der Vergangenheit zugetragen hat, sondern legen sich zunächst auf Regeln in Bezug darauf fest, welche Attribute Quellen aufweisen müssen, um konsultationswürdig zu sein, und welche Arten von Quellen im Zweifelsfall gegenüber konfligierenden Quellen vorzuziehen sind. ${ }^{20}$

Weiter oben habe ich geschrieben, während die Verwendungsweise von »trocken« kriterial mit der Frage verbunden sei, ob der Mars zu jedem Zeitpunkt entweder trocken oder nicht trocken war, seien Zeugenaussagen und sprachliche Quellen nur symptomatisch (oder evidenziell) mit den untersuchten Vorfällen respektive geschichtlichen Ereignissen verbunden. Wenn wir unseren Fokus statt auf die jeweils relevanten sprachlichen Evidenzen auf die Meta-Erwägungen richten, die in den drei Fällen angestellt werden müssen, befinden sich der Gerichts- und der Historiker-Fall näher beim Mars-Fall: Die Regeln in Bezug darauf, unter welchen Bedingungen eine Zeugenaussage als zuverlässig gilt oder unter welchen Bedingungen welche Rückschlüsse aus der Existenz eines bestimmten historischen Dokuments gezogen werden können, sind nicht evidenziell, sondern kriterial dafür, wie wir die Frage beantworten sollten, ob Smith Jones umgebracht hat und ob sich dieses-und-dieses Ereignis

19 Vgl. Williamson 2007, S. 4of. Zu den erwähnten Logiken und ihren Implikationen für den Mars-Fall: S. 31-36.

20 Vgl. Williamson 2007, S. 42. Er bespricht diesen Punkt allerdings nur andeutungsweise. 
zugetragen hat oder nicht. Dennoch besteht auch in Bezug auf die Rolle, die Meta-Erwägungen jeweils spielen, ein entscheidender Unterschied zwischen dem Mars-Fall und den Gerichts- und Historiker-Fällen: In den Gerichts- und den Historiker-Fällen haben wir es jeweils mit zwei unterschiedlichen Fragen zu tun:

a. Hat Smith Jones umgebracht?

b. Sollten wir angesichts unserer Evidenz sagen, dass Smith Jones umgebracht hat?

$a^{\prime}$. Was ist zu einem bestimmten historischen Zeitpunkt passiert?

$\mathrm{b}^{\prime}$. Was sollten wir angesichts unserer Evidenz darüber sagen, was zu dem betreffenden historischen Zeitpunkt passiert ist?

Nur $b$ beziehungsweise $b^{\prime}$ stehen zu den oben beschriebenen Meta-Erwägungen in einem kriterialen Zusammenhang. Dies gilt im Mars-Fall nicht:

a". War der Mars zu jedem Zeitpunkt entweder trocken oder nicht trocken?

b". Sollten wir angesichts unserer Evidenz sagen, dass der Mars zu jedem Zeitpunkt entweder trocken oder nicht trocken war?

Hier sind die Meta-Erwägungen darüber, ob beispielsweise der Satz (7) logisch widersprüchlich ist, nicht nur für die Beantwortung von $b^{\prime \prime}$, sondern auch für die Beantwortung von a" kriterial. Und wenn wir die eingangs beschriebenen Umstände über das Flüssigkeitsvorkommen auf dem Mars voraussetzen, läuft $a^{\prime \prime}$ auf exakt dieselbe Frage hinaus wie b".

Mit dem im vorangehenden Absatz diskutierten Unterschied zwischen dem Mars-Fall und den Gerichts- und Historiker-Fällen hängt eine weitere Ungleichheit der Fälle zusammen: Im Mars-Fall könnten wir, wie Williamson selbst einräumt, über sämtliche relevanten empirischen Informationen verfügen und dennoch würde uns die Beantwortung der Frage (1) Kopfzerbrechen bereiten. Um sie zu beantworten, müssen wir uns mit den oben genannten Fragen zum Funktionieren des Prädikats »ist trocken« oder zum Funktionieren vager Prädikate im Allgemeinen auseinandersetzen. Im Kontrast dazu würden sich die oben genannten Meta-Erwägungen, die mit den Fragen des Gerichts und den Fragen von Historikerinnen und Historikern assoziiert sind, überhaupt nicht stellen, wenn wir über vollständige Evidenz verfügten: Wenn wir genau wüssten, was Smith getan hat und was sich zu welchem historischen Zeitpunkt zugetragen hat, müssten wir nicht auf Zeugenaussagen oder historische Quellen zurückgreifen und folglich wäre es für uns auch nicht von Belang, unter welchen Bedingungen sie zuverlässig sind respektive welche Aussagekraft ihnen zukommt.

Alles in allem sollte anhand der vorangehenden Ausführungen deutlich geworden sein, dass der Mars-Fall hinsichtlich der Rolle, die sprachliche Ausdrücke und metasprachliche Erwägungen in der Auseinandersetzung mit 
ihm spielen, ganz anders geartet ist als der Gerichts- und der Historiker-Fall und dass es Gründe dafür gibt, zu behaupten, dass (1) von sprachlichen Ausdrücken oder Begriffen handelt, die nicht gleichermaßen dafür angeführt werden können, Fragen, wie sie vom Gericht oder von Historikerinnen und Historikern gestellt werden, als Fragen über sprachliche Ausdrücke oder Begriffe zu betrachten.

\subsection{Alternative empirische Umstände}

Unter den in 2.2 geschilderten Umständen müssen wir für die Beantwortung der Frage (1) auf sprachlich-begriffliche Erwägungen zurückgreifen. Dies könnte, wie bereits erwähnt, als Argument dafür angeführt werden, dass (1) eine begriffliche Frage ist. Allerdings sind alternative empirische Umstände denkbar, unter denen es für die Beantwortung von (1) nicht erforderlich ist, über das Funktionieren von »trocken« in Grenzfällen nachzudenken. Hätte es zum Beispiel auf dem Mars zu keinem Zeitpunkt auch nur ein einziges Molekül einer Flüssigkeit gegeben, dann wäre die Frage (1) eindeutig zu bejahen: Der Mars war zu jedem Zeitpunkt trocken, also war er zu jedem Zeitpunkt entweder trocken oder nicht trocken. ${ }^{21}$ Im Lichte dieser Erkenntnis erscheint es problematisch, daran festzuhalten, dass (1) eine begriffliche Frage ist.

Für die Zwecke der vorliegenden Abhandlung ist es jedoch nur relevant, dass der Satz (1) unter den zu Beginn des Abschnitts 2.2 beschriebenen Bedingungen eine begriffliche Frage ausdrückt. Je nachdem, in welchem Kontext (1) formuliert wird, haben wir es eben mit unterschiedlichen Fragen zu tun: (1) kann als empirische Frage gestellt werden in einem Kontext, in welchem die Sprecherin nicht weiß, wie es sich mit den Flüssigkeitsmolekülen auf dem Mars verhält und verhalten hat. Für einen solchen Fall ist die Formulierung der Frage allerdings seltsam. Natürlicher wäre die Frage: »Ist es auf dem Mars trocken?«. Und wenn diese Frage mit $» j a$ « beantwortet wird, könnte man weiterfragen: »Und war es immer schon trocken?«. Direkt einzusteigen mit »War es auf dem Mars zu jedem Zeitpunkt entweder trocken oder nicht trocken?« ist für jemanden, der sich für das Flüssigkeitsvorkommen auf dem Mars interessiert, keine naheliegende Formulierung. Aber natürlich ist es prinzipiell möglich, mit (1) diese empirische Frage zum Ausdruck zu bringen. ${ }^{22}$

\footnotetext{
21 Vgl. Williamson 2007, S. 25.

22 Man könnte unter diesen Umständen allerdings trotzdem versuchen, dafür zu argumentieren, dass man die Frage nicht wirklich versteht, wenn man nicht auch die begriffliche Frage beantworten könnte, unter welchen Bedingungen sie zu bejahen oder zu verneinen wäre.
} 
In Kontexten, in welchen eine bestimmte Annahme darüber vorausgesetzt wird, wie es sich mit den Flüssigkeitsmolekülen auf dem Mars verhält und verhalten hat, drückt die Frage (1) eine begriffliche Frage aus: Ist bzw. wäre es unter den-und-den Bedingungen korrekt, den Mars so-und-so zu beschreiben? In diesem Fall gehören die jeweiligen Bedingungen zur Frage. Wir fragen zum Beispiel:

(8) Angenommen, es gab nie ein einziges Flüssigkeitsmolekül auf dem Mars: War es dann auf dem Mars zu jedem Zeitpunkt entweder trocken oder nicht trocken?

Oder wir fragen:

(9) Angenommen, es gab früher einmal sehr viel Wasser auf dem Mars, und dieses Wasser sei im Laufe der Zeit sukzessive verdunstet: War es dann auf dem Mars zu jedem Zeitpunkt entweder trocken oder nicht trocken? Dass (8) eine andere Frage als (9) ist, ist offensichtlich. (8) ist philosophisch auch deutlich weniger interessant als (9), und für die Beantwortung von (8) müssen wir uns, wie oben bereits erwähnt, nicht mit der Debatte um das Thema Vagheit auseinandersetzen. Da (1) unter verschiedenen Umständen unterschiedliche Fragen ausdrückt, folgt daraus, dass eine von (1) ausgedrückte Frage unter bestimmten Bedingungen ohne Rekurs auf begriffliche Erwägungen beantworten werden kann, nicht, dass es nicht andere Bedingungen (wie eben beispielsweise die eingangs beschriebenen) gibt, untern denen (1) eine begriffliche Frage ausdrückt.

\subsection{Ein Streit um Worte?}

Handelt (1) - in einem Kontext, in welchem der Fragesatz eine begriffliche Frage ausdrückt - vom Mars (und von Trockenheit) oder von Wörtern oder Begriffen? Man könnte geneigt sein, zu behaupten, dass an dieser Entscheidung nicht viel hänge. »der Mars « steht an Subjektstelle des Fragesatzes, und in vielen Fällen besteht eine natürliche Weise, die Frage zu beantworten, wovon ein Satz handle, darin, das Satzsubjekt anzugeben. ${ }^{23}$ Andererseits ist für die Beantwortung von (1) unter den eingangs beschriebenen Bedingungen, wie bereits erwähnt, keine Untersuchung des Mars erforderlich, sondern einzig eine Analyse unseres Sprachgebrauchs beziehungsweise unserer Begriffe, was für die Behauptung sprechen würde, der Satz handle entgegen dem, was seine Oberflächenstruktur nahelegt, von der Sprache oder von Begriffen. Da »handeln von« beziehungsweise auch das englische »to be about« relativ vage Ausdrücke sind, ergibt es nicht viel Sinn, darauf zu bestehen, dass die Frage vom Mars handle oder dass sie von Sprache oder Begriffen handle.

23 Vgl. dazu auch den Topik-Begriff der Linguistik. Siehe z. B. Bußmann 2008. 
Die Entscheidung, ob philosophische Fragen und philosophische Aussagen von der Sprache oder von der Welt handeln, erscheint auch in anderen Zusammenhängen müßig. Wenn wir uns mit den notwendigen und hinreichenden Bedingungen für das Verfügen über Wissen befassen: Befassen wir uns dann mit dem Begriff des Wissens, mit der Verwendungsweise des Wortes »Wissen« oder mit dem entsprechenden »Phänomen«? Handelt die Frage, was Überzeugungen sind, von Überzeugungen oder vom Begriff der Überzeugung? Und befasst sich die Philosophie des Geistes mit dem Geist oder mit der etablierten Gebrauchsweise von »Geist«? In diesen Kontexten schließen die einen Varianten die anderen jeweils nicht aus, im Gegenteil: Indem wir uns mit dem Gebrauch von »Wissen«, »Überzeugung« und »Geist« (oder deren Äquivalenten in anderen Sprachen) befassen, befassen wir uns auch mit dem Begriff des Wissens, dem Begriff der Überzeugung und dem Begriff des Geistes - und mit Wissen, mit Überzeugungen und mit dem Geist. ${ }^{24}$

Dies bedeutet nicht, dass das Wort »Wissen « mit Wissen identisch ist oder der Begriff des Geistes dasselbe ist wie der Geist. »Wissen« ist im Gegensatz zu Wissen aus Buchstaben zusammengesetzt, und während die Idee, Wissen sei wahre, gerechtfertigte Überzeugung, prima facie eine gewisse Plausibilität hat, wäre es völlig absurd, zu behaupten, der Begriff des Wissens sei wahre, gerechtfertigte Überzeugung. Wir können außerdem einen Teil unseres Wissens verlieren, ohne einen Teil unseres Wissensbegriffs zu verlieren. Analog dazu ist der Geist offensichtlich nicht mit dem Wort »Geist« oder dem Begriff Geist identisch. »Geist« ist im Gegensatz zum Geist Teil der deutschen Sprache, und es gibt Wesen, die einen Geist besitzen, ohne den Begriff des Geistes zu besitzen.

Dass wir uns, indem wir uns mit der Verwendung von »Wissen« befassen, ipso facto auch mit dem Begriff Wissen und mit Wissen befassen, bedeutet außerdem auch nicht, dass es keine Untersuchung davon, was Wissen ist, geben kann, die nicht gleichzeitig eine Klärung des Wissens-Begriffs oder der Bedeutung des Wortes »Wissen« ist. NaturwissenschaftlerInnen, die erforschen, wie Wissen physikalisch realisiert ist beziehungsweise neuronal »repräsentiert « wird, ${ }^{25}$ setzen sich beispielsweise auch in einem bestimmten Sinn damit auseinander, was Wissen ist, aber sie betreiben keine Begriffsanalyse. Die philosophische Untersuchung davon, was Wissen ist, besteht jedoch der Position zufolge, die im vorliegenden Buch verteidigt wird, in nichts anderem als der Klärung des Begriffs des Wissens und seinen Relationen zu

24 Vgl. Hanfling 2000, S. 15-18, Hacker 2009b, S. 13.

25 Für eine Kritik an der Rede von mentalen Repräsentationen vgl. z. B. Hacker 1987, S. 497499, Glock 2006, S. 46-48. 
anderen Begriffen. Und dasselbe gilt für die philosophischen Untersuchungen davon, was Überzeugungen sind, was der Geist ist und für viele andere Was-ist$\mathrm{X}$-Fragen, zu denen in der theoretischen Philosophie Debatten geführt werden.

In bestimmten Kontexten hängt also nicht viel davon ab, ob wir sagen, dass philosophische Probleme von Wörtern oder von Begriffen handeln, oder stattdessen sagen, sie handeln von den entsprechenden »Phänomenen«. Im Widerstreit zwischen der hier vertretenen Auffassung und derjenigen von Williamson geht es aber trotzdem nicht nur um unterschiedliche präferierte Formulierungen beziehungsweise eine unterschiedliche Gewichtung der Frage, wovon philosophische Probleme handeln. Williamson würde nämlich der im vorangehenden Absatz charakterisierten Position über das Verhältnis von philosophischen Was-ist-X-Fragen mit Fragen nach den entsprechenden Begriffen nicht zustimmen. Für ihn läuft beispielsweise die philosophische Frage danach, was Wissen ist, nicht auf dasselbe hinaus wie die Frage nach der korrekten Analyse unseres Wissensbegriffs. Wofür wir uns in der Philosophie interessieren, so Williamson, ist die Natur von Wissen. In seinem Kapitel »Thought Experiments« lesen wir dazu: Wenn sich herausstellt, dass es begrifflich möglich ist, die wahre, gerechtfertigte Überzeugung, dass $p$, zu besitzen, ohne zu wissen, dass $p$, dann ist damit noch nicht die epistemologisch interessantere Frage beantwortet, ob es auch metaphysisch möglich ist, über wahre, gerechtfertigte Überzeugung ohne Wissen zu verfügen. Begriffliche $\mathrm{Zu}-$ sammenhänge werden damit nicht bedeutungslos für die Philosophie: Wäre der Begriff der wahren, gerechtfertigten Überzeugung mit dem Begriff des Wissens identisch, so Williamson, so wäre damit »vermutlich « (presumably) auch entschieden, dass die Natur von wahrer, gerechtfertigter Überzeugung mit der Natur von Wissen identisch wäre. Aber in erster Linie wollen wir in der Epistemologie Erkenntnisse über die Natur von Wissen gewinnen, und diese ist es auch, über welche uns philosophische Gedankenexperimente Aufschluss geben:Was wir anhand unserer Auseinandersetzung mit Gettier-Fällen herausfinden, ist beispielsweise, dass es metaphysisch möglich ist, wahre, gerechtfertigte Überzeugung ohne Wissen zu besitzen. ${ }^{26}$

Im Rahmen des vorliegenden Buchs kann ich nicht ausführlich auf den vermeintlichen Unterschied zwischen begrifflicher und metaphysischer Notwendigkeit eingehen. Ich teile die Urteile oder »Intuitionen« in Bezug auf Gedankenexperimente $\grave{a}$ la Kripke und Putnam nicht, die zur gegenwärtig populären Ausprägung dieser Unterscheidung Anlass gegeben haben und habe anderswo dafür argumentiert, dass wir es, wenn wir zu sagen geneigt sind, »Wasser ist $\mathrm{H}_{2} \mathrm{O}$ « respektive »Wasser ist trinkbar« seien jeweils in einem

Vgl. Williamson 2007, S. 206. 
bestimmten Sinn notwendig, in einem anderen aber kontingent, nicht mit zwei verschiedenen Arten von Notwendigkeit, sondern mit zwei verschiedenen Wasser-Begriffen zu tun haben: Wenn wir einen alltäglichen Wasser-Begriff zugrunde legen, so könnte man argumentieren, ist »Wasser ist trinkbar« notwendig und »Wasser ist $\mathrm{H}_{2} \mathrm{O}$ « kontingent. Wenn wir einen wissenschaftlichen Wasser-Begriff zugrunde legen, ist »Wasser ist $\mathrm{H}_{2} \mathrm{O}$ « notwendig und »Wasser ist trinkbar« kontingent. Gemeint ist aber immer begrifflich notwendig und begrifflich kontingent. ${ }^{27}$ Was die Natur von Wissen anbelangt, stimme ich Wittgensteins Diktum zu, die Natur (beziehungsweise das »Wesen«) sei in der Grammatik ausgesprochen:28 Wenn wir überhaupt von so etwas wie der Natur von Wissen sprechen wollen, dann wird diese Natur durch unseren Sprachgebrauch und den sich daraus ergebenden Begriff des Wissens bestimmt.

\subsection{Substanzielle Wahrheiten über die Welt}

Mit Williamsons Konzeption von metaphysischen Möglichkeiten und Notwendigkeiten hängt ein weiterer wichtiger Aspekt zusammen, in welchem er von der im vorliegenden Buch verteidigten Auffassung davon abweicht, worin das Unterfangen der Philosophie besteht. Williamson prüft das folgende Argument dafür, dass die Philosophie von Begriffen handelt: ${ }^{29}$ Philosophie wird im Lehnstuhl betrieben. Aus dem Lehnstuhl können wir aber keine substanziellen Wahrheiten über die Welt erkennen. Damit Philosophie überhaupt erfolgreich betrieben werden kann, muss ihr Unterfangen also in etwas anderem als dem Versuch bestehen, substanzielle Wahrheiten über die Welt zu erkennen. Und als naheliegender alternativer Kandidat kommt in Betracht, dass sie mit der Untersuchung von Begriffen befasst ist. Williamson bestreitet die zweite Prämisse dieses Arguments. Im Kapitel zu der Frage, wovon philosophische Probleme handeln, sagt er zunächst einmal nur, dass es nicht selbstverständlich ist, dass man aus dem Lehnstuhl keine substanziellen Wahrheiten über die Welt erkennen kann:

[W] e should be open to the idea that thinking just as much as perceiving is a way of learning how things are. Even if one does not fully understand how thinking can provide new knowledge, the cases of logic and mathematics constitute overwhelming evidence that it does so. [...] [C]onceptions of logic and mathematics as [...] somehow trivial or non-substantial have not been vindicated by any clear explanation of the relevant sense of "trivial" or "non-substantial." 30

27 Vgl. Rathgeb 2016.

28 Vgl. $P U \S 371$.

29 Ein ähnliches Argument (von Severin Schroeder) wurde im Kapitel 1 des vorliegenden Buchs besprochen.

3o Williamson 2007 , S. 47 . 
An späterer Stelle argumentiert er dann explizit dafür, dass unser Wissen über gewisse metaphysische Möglichkeiten und Notwendigkeiten zu einer Kategorie von Lehnstuhl-Wissen gehört, das weder eindeutig a priori noch $a$ posteriori ist und im Falle dessen es uns nicht überraschen sollte, wenn es sich unter anderem auch über Wahrheiten über die externe Welt erstreckt. ${ }^{31}$

Ich habe bereits weiter oben dafür argumentiert, dass es innerhalb des Bereichs, den wir als »philosophisches Wissen« bezeichnen können, zwei Kategorien zu unterscheiden gilt:

A) Wissen über komplexe begriffliche Wahrheiten wie

- Überzeugungen befinden sich nicht im Gehirn.

- Es kann keine private Sprache geben.

- Wesen, die keine Sprache besitzen, können nur solche mentalen Eigenschaften besitzen, die sich in nonverbalem Verhalten manifestieren können.

B) Wissen über Wahrheiten, die sich aus einer Kombination begrifflicher Wahrheiten mit offensichtlichen empirischen Wahrheiten ergeben, wie

- Menschen handeln manchmal aus freiem Willen.

- Es gibt Dinge, die wir wissen.

- Tiere können nur solche mentalen Eigenschaften besitzen, die sich in nonverbalem Verhalten manifestieren können. (Diese Wahrheit ergibt sich aus dem dritten Beispiel aus der Kategorie A zusammen mit der (teilweise) empirischen Wahrheit, dass Tiere keine Sprache besitzen. ${ }^{32}$ )

Mindestens die ersten zwei Beispiele der Kategorie B handeln von der externen Welt, haben aber, genau wie das dritte B-Beispiel und die Beispiele der Kategorie A, nichts mit metaphysischer Möglichkeit oder Notwendigkeit zu tun (so etwas gibt es meines Erachtens, wie bereits erwähnt, nicht) und siedeln sich auch nicht in einem Graubereich zwischen A priori- und A posteriori-Wissen an. Handelt die Aussage, dass sich Überzeugungen nicht im Gehirn befinden, von der Welt? Aufgrund der grammatischen Form ist es auch hier wieder naheliegend, zu sagen, dass die Aussage von Überzeugungen handelt. Und da Überzeugungen in einem bestimmten Sinn Teil der Welt sind (es gibt in der Welt Personen mit Überzeugungen), könnte man dann auch sagen, dass die Aussage von der Welt oder von Dingen (in einem weiten Sinn) in der Welt handelt.

31 Vgl. Williamson 2007, S. 169.

32 Auch dass Tiere keine Sprache besitzen, ist wieder eine philosophisch kontroverse Aussage, die unter anderem auf der Grundlage von begrifflichen Erwägungen darüber getroffen werden muss, unter welchen Bedingungen man einem Wesen eine Sprache zuschreiben kann. 
Andererseits steht die Aussage »Überzeugungen befinden sich nicht im Gehirn « in einem ganz anderen Verhältnis zur Welt und zu (Personen mit) Überzeugungen als »Karpfen leben nicht in salzigem Gewässer« zu Karpfen steht. ${ }^{33}$ Der Grund hierfür besteht nicht einfach darin, dass Überzeugungen im Gegensatz zu Karpfen keine raum-zeitlichen Gegenstände sind, ${ }^{34}$ sondern insbesondere auch darin, dass die erste Aussage begrifflich und somit notwendig wahr ist. Während es eine Welt geben könnte, in der Karpfen in Salzwasser leben, könnte es keine Welt geben, in der Überzeugungen sich im Gehirn befinden. Die Wahrheit, dass Überzeugungen sich nicht im Gehirn befinden, ist deshalb gewissermaßen unabhängig von den Tatsachen in der Welt. Dass »Überzeugungen befinden sich nicht im Gehirn" wahr ist, ergibt sich allein aus den Bedeutungen der Wörter, aus denen der Satz zusammengesetzt ist, und der Weise ihrer Kombination und ist insofern im Gegensatz zu »Karpfen leben nicht in salzigem Gewässer « keine Wahrheit über die Welt - keine Wahrheit, die von der Welt handelt.

Gegen die im vorangehenden Absatz zum Ausdruck gebrachte Auffassung könnte man allerlei Einwände vorbringen, die zum Beispiel damit zusammenhängen, dass die Wahrheit begrifflicher Wahrheiten mit kontingenten Tatsachen darüber zusammenzuhängen scheint, wie wir sprachliche Ausdrücke verwenden, und dass mithin doch eine Welt möglich zu sein scheint, in welcher »Überzeugungen sind nicht im Gehirn « unwahr ist. ${ }^{35}$ Statt diese Punkte zu besprechen, möchte ich jedoch auf die allgemeineren Bedenken im Zusammenhang mit begrifflichen oder analytischen Wahrheiten eingehen, die Williamson im dritten und vierten Kapitel seines Buchs vorbringt. Vorher allerdings noch ein Wort zu Williamsons Bezugnahme auf logisches und mathematisches Wissen in der oben (S. 177) zitierten Passage. Williamson schreibt dort, die Fälle der Logik und der Mathematik seien gute Beispiele dafür, dass man durch bloßes Nachdenken - also ohne empirische Forschung - neues Wissen gewinnen könne, von dem bisher nicht plausibel gemacht werden konnte, dass es trivial oder nicht-substanziell sei.

33 Vgl. dazu auch Baker/Hacker 2009, S. 280-283.

34 Man könnte ähnlich auch mit Bezug auf »Computer können nicht denken« anstelle von »Überzeugungen sind nicht im Gehirn« argumentieren, und Computer sind raumzeitliche Gegenstände. Der Grund dafür, weshalb ich nicht das Computer-Beispiel gewählt habe, besteht darin, dass in diesem Fall mehr Diskussionsspielraum in Bezug darauf besteht, ob die Eigenschaften, die dafür ausschlaggebend sind, dass Computer nicht denken können, sich tatsächlich aus dem Begriff des Computers ergeben.

35 Diesen spezifischen Einwand habe ich bereits (samt einer entsprechenden Replik) im Abschnitt 2.1 des vorliegenden Kapitels vorweggenommen. 
Mit neues Wissen, nicht trivial und substanziell werden hier meines Erachtens drei Kategorien vermischt, die nicht in der suggerierten Weise zusammenhängen. Williamson argumentiert in der betreffenden Passage dafür, dass daraus, dass wir philosophisches Wissen aus dem Lehnstuhl gewinnen, nicht folge, dass dieses Wissen kein Wissen über die Welt sei. Die Logik und die Mathematik wären in diesem Kontext nur für Williamsons Punkt einschlägige Beispiele, wenn in diesen Bereichen aus dem Lehnstuhl Wissen über die Welt erlangt würde. Und das kann offensichtlich bestritten werden. ${ }^{36}$ Zwar sind die Logik und die Mathematik Lehnstuhl-Disziplinen, aber in Bezug auf ihre Weltbezogenheit scheint es um sie doch ebenso bestellt zu sein wie mit dem oben besprochenen Beispiel »Überzeugungen befinden sich nicht im Gehirn«. Auch die Wahrheiten der Logik und der Mathematik sind notwendig, hängen also nicht davon ab, was der Fall ist, und handeln insofern nicht von der Welt. In diesem Sinn wäre es meines Erachtens auch richtig, sie als nicht-substanziell zu bezeichnen. Dass wir in der Logik und der Mathematik ebenso wie in der Philosophie neues Wissen gewinnen können und dass es in allen drei Bereichen nicht-triviale Wahrheiten gibt, hat mit der Frage, ob diese Wahrheiten substanziell sind, nichts zu tun. ${ }^{37}$ Keine der oben unter (A) genannten Wahrheiten ist trivial: Die Wahrheit dieser Sätze ist nicht offensichtlich (tatsächlich wird die Wahrheit aller drei Sätze von vielen Philosophinnen und Philosophen bestritten), und es ist einiges an Erklärungsaufwand damit verbunden, diese Urteile als wahr zu erweisen.

Da ich von der Wahrheit vieler philosophischer Urteile - zum Beispiel davon, dass es keine private Sprache geben kann - zunächst überzeugt werden musste, finde ich es auch adäquat, zu behaupten, dass in der Philosophie neues Wissen gewonnen wird. Ich wusste früher nicht, dass es keine private Sprache geben kann, und weiß es heute. Auch dies hat nichts mit der Frage zu tun, ob das Urteil, dass es keine private Sprache geben kann, von der Welt handelt oder nicht. Dass auch in der Logik und in der Mathematik neues, nichttriviales Wissen erlangt werden kann, zeigt also nicht, dass es möglich ist, aus dem Lehnstuhl Wissen über die Welt zu erlangen. Und was es bedeutet, dass logische und mathematische Wahrheiten nicht-substanziell sind, ist meines Erachtens pace Williamson ziemlich klar: Es bedeutet eben, dass sie nicht davon handeln, was in der Welt der Fall ist (vgl. dazu allerdings auch die Diskussion weiter unten, in 3.2).

36 Vgl. z. B. Glock 2010, S. 341.

37 Dass daraus, dass eine Wahrheit trivial ist, nicht folgt, dass sie nicht-substanziell ist, räumt Williamson an anderer Stelle selbst ein. Vgl. Williamson 2007, S. 65. 


\subsection{Einschränkung der Fragestellung auf die Kernwahrheiten der Philosophie}

In den Kapiteln 3 und 4 seiner Monographie befasst sich Williamson mit der Frage, ob philosophische Wahrheiten, auch wenn sie nicht von Begriffen handeln, vielleicht dennoch im Allgemeinen begrifflich wahr sein könnten. »Begrifflich wahr« verwendet er dabei gleichbedeutend mit »analytisch«,38 was P. M.S. Hacker in seiner Besprechung von Williamson kritisiert: Unter den Philosophinnen und Philosophen, die die Philosophie als ein rein begriffliches Unterfangen betrachtet haben, habe es, so Hacker, nur einen einzigen gegeben, der die Auffassung vertreten habe, dass alle philosophischen Aussagen analytisch seien, nämlich A. J. Ayer. Und Aussagen wie »Es kann keine private Sprache geben« oder »Der Idealismus und der Materialismus sind zwei Antworten auf eine fehlgeleitete Frage « seien begrifflich wahr, aber nicht analytisch. ${ }^{39}$ Ich verwende im vorliegenden Buch »analytisch « im weiten Sinn von »wahr aufgrund von Bedeutung« (zu dieser Formulierung weiter unten mehr) und lege deshalb im Gegensatz zu Hacker keinen Wert auf die Unterscheidung zwischen »begrifflich wahr« und »analytisch«. Aber natürlich gibt es einen großen Unterschied zwischen den beiden Begriffen, wenn wir »analytisch« in einem kantischen oder fregeanischen Sinn gebrauchen.

Williamson setzt sich also mit der Frage auseinander, ob philosophische Wahrheiten analytisch sind, und meint schon gleich zu Beginn seiner Besprechung dieser Frage, dass dies auf jeden Fall nicht für philosophische Wahrheiten im Allgemeinen gelten könne:

Many philosophically relevant truths are clearly not conceptual truths in any
useful sense. For instance, in arguing against subjective idealism, a defender of
common sense metaphysics says that there was a solar system millions of years
before there was sentient life. Similarly, a defender of common sense epistemol-
ogy says that he knows that he has hands; that he knows that he has hands is no
conceptual truth [...]. Philosophers of mind and language dispute whether there
is a language of thought; whatever the answer, it is no conceptual truth. Natural-
ists and anti-naturalists dispute whether there is only what there is in space and
time; again, the answer is unlikely to be a conceptual truth. ${ }^{40}$

Williamson spricht in dem betreffenden Abschnitt nicht von »philosophical truths«, sondern von »philosophically relevant truths«, und führt dabei zum

38 Vgl. Williamson 2007, S. 50.

39 Vgl. Hacker 2009a, S. 343.

40 Williamson 2007 , S. 49. 
einen die folgenden zwei empirischen Wahrheiten an, die ihm zufolge im $\mathrm{Zu}$ sammenhang mit philosophischen Fragen angeführt werden können:

- Es gab schon Millionen von Jahren bevor es denkende Wesen gab, ein Sonnensystem.

- Ich weiß, dass ich Hände habe.

Diese Aussagen sind natürlich keine begrifflichen Wahrheiten, man könnte sich aber auch darüber streiten, ob sie sinnvollerweise in der von Williamson behaupteten Weise in Diskussionen der philosophischen Fragen eingehen sollten, ob etwas außerhalb des Geistes existiert und ob wir etwas wissen können. Dies zu entscheiden, ist im vorliegenden Kontext nicht nötig, da wir uns ja nur dafür interessieren, ob philosophische Wahrheiten begrifflich wahr sind. Die obigen beiden Beispiele sind keine philosophischen Wahrheiten.

Zum anderen führt Williamson in der oben zitierten Passage auch philosophische Fragen an, im Falle derer er es für offensichtlich (oder jedenfalls sehr plausibel) zu halten scheint, dass ihre korrekten Antworten keine begrifflichen Wahrheiten sind (zumindest behauptet er dies, ohne dafür zu argumentieren): - Gibt es eine Sprache des Geistes?

- Existiert nur das, was in Raum und Zeit existiert?

Wittgensteins Argument dafür, dass eine Sprache des Geistes unmöglich ist (was natürlich die Nicht-Existenz einer Sprache des Geistes impliziert), ist aber ein Argument, das allein auf die Begriffe der Sprache, des Geistes und damit zusammenhängende Begriffe abstellt. Und es wäre auch wenigstens denkbar, dass eine Analyse des Begriffs der Existenz ergibt, dass »Existenz« dasselbe wie »Existenz in Raum und Zeit« bedeutet. In diesem Fall könnte man auch die zweite Frage durch eine begriffliche Wahrheit beantworten.

Williamson schließt auf der Grundlage der genannten Beispiele, dass ohnehin nur zur Debatte stehen kann, ob ein zentraler Kern philosophischer Wahrheiten begrifflich wahr ist, und nicht, dass philosophische Wahrheiten generell begrifflich wahr sind. Mit dieser Konklusion bin ich, wie oben in 2.6 dargelegt, einverstanden, auch wenn ich Williamsons Argumentation dafür aus den oben angegebenen Gründen als nicht stichhaltig erachte. Ich gehe deshalb nachfolgend zu Williamsons Argumenten gegen die Auffassung über, dass wenigstens die Kernwahrheiten der Philosophie begrifflich wahr sind.

\subsection{Frege-Analytizität}

In seiner Besprechung des Themas Analytizität befasst sich Williamson zunächst mit so genannt »metaphysischen « Konzeptionen von Analytizität. Diese Konzeptionen definiert er so, dass ihnen zufolge ein Urteil genau dann analytisch ist, wenn es wahr ist unabhängig davon, wie die Welt ist. Aus Gründen, auf die ich weiter unten eingehen werde, lehnt Williamson die Idee 
ab, dass es solche Urteile gibt. Im betreffenden Kapitel seiner Monographie bespricht Williamson aber auch Konzeptionen von Analytizität, die man im Anschluss an Paul Boghossian (1997) als »semantisch « bezeichnen könnte, unter anderem Gottlob Freges Konzeption von Analytizität. Nach dieser Konzeption ist ein Satz genau dann analytisch, wenn man ihn in eine logische Wahrheit überführen kann, indem man einen oder mehrere seiner Teilausdrücke durch ein Synonym ersetzt. »Alle Erpel sind männliche Enten« ist zum Beispiel Frege-analytisch, weil wir, indem wir für »männliche Enten« den synonymen Ausdruck »Erpel« einsetzen, eine logische Wahrheit erhalten: »Alle Erpel sind Erpel«. Williamson zufolge ist dies eine völlig respektable Definition von Analytizität. Allerdings können wir unter Rückgriff auf Frege-Analytizität Williamson zufolge keinen philosophischen Exzeptionalismus begründen. Denn erstens wäre es aussichtslos, zeigen zu wollen, dass die Kernwahrheiten der Philosophie Frege-analytisch sind. ${ }^{41}$ Und zweitens, selbst wenn gezeigt werden könnte, dass alle philosophischen Wahrheiten Frege-analytisch sind, würde dies für sich allein weder einen philosophischen Exzeptionalismus mit Bezug auf die Methode noch einen Exzeptionalismus mit Bezug auf den Gegenstandsbereich der Philosophie begründen.

Zunächst zur Methode: Dass ein Satz Frege-analytisch ist, so Williamson, sagt für sich allein nichts darüber aus, wie wir ihn wissen können. Selbst wenn wir zeigen könnten, dass sprachkompetente Personen ohne Weiteres dazu in der Lage sind, Frege-analytische Sätze auf die entsprechenden logischen Wahrheiten zurückzuführen (diese Idee ist Williamson zufolge schon per se unplausibel), stellte sich die Frage, worauf sich dann ihr Wissen über logische Wahrheiten gründet und ob sie dieses tatsächlich auf ganz andere Weise erlangen als empirisches Wissen. ${ }^{42}$

Dann zum Gegenstandsbereich: Dass ein Satz Frege-analytisch ist, zeigt Williamson zufolge nicht, dass er in irgendeinem Sinn nicht-substanziell ist. Philosophinnen und Philosophen, die glauben, dass analytische Sätze nichtsubstanziell seien, erläutern diese Behauptung Williamson zufolge bisweilen damit, dass die Wahrheit dieser Sätze keine echten Anforderungen an die Welt stellt oder dass die Sätze keinen echten Fall ausschließen. Aber weshalb, fragt Williamson, sollten wir nicht sagen, dass Frege-analytische oder logische Wahrheiten einen echten Fall ausschließen? Die Wahrheit, dass alle Erpel Erpel sind, schließt zum Beispiel den Fall aus, dass einige Erpel keine Erpel sind. Wenn wir, um solche Beispiele abzuwehren, darauf insistieren, dass eine Wahrheit, um substanziell zu sein, einen logisch konsistenten Fall ausschließen

41 Vgl. Williamson 2007 , S. $68 \mathrm{f}$.

42 Vgl. Williamson 2007, S. 66-68. 
muss, dann haben wir, so Williamson, nicht erfolgreich dafür argumentiert, dass logische Wahrheiten keinen Fall ausschließen, sondern dies einfach stipuliert: es durch die Hintertür zu einer Definitionssache gemacht. ${ }^{43}$

Williamson hat Recht damit, dass weder philosophische Wahrheiten im Allgemeinen noch eine Gruppe von philosophischen Kernwahrheiten Fregeanalytisch sind. Aber seine Argumentation dafür, dass selbst dann kein philosophischer Exzeptionalismus folgen würde, wenn alle philosophischen Wahrheiten begrifflich auf logische Wahrheiten zurückführbar wären, ist nicht überzeugend. Tatsächlich erlangen wir unser Wissen über Frege-analytische oder über logische Wahrheiten auf andere Weise als unser Wissen über empirische Wahrheiten, nämlich in der Regel durch bloßes Verstehen (vgl. die Diskussion im Abschnitt 4 des vorliegenden Kapitels). Und dass logische Wahrheiten keinen Fall ausschließen und mithin nicht-substanziell sind, ist tatsächlich eine definitorische Wahrheit. Es ergibt sich daraus, was es bedeutet, einen echten Fall auszuschließen. Echte Fälle sind logisch-begrifflich konsistent, sie stellen eine Weise dar, wie die Welt auch sein könnte. Und die Welt könnte nicht auch so sein, dass einige Erpel keine Erpel sind. ${ }^{44}$

Dass mitunter der Eindruck entstehen kann, dass logisch-begriffliche Wahrheiten doch einen Fall ausschließen, beruht darauf, dass die Sätze, die sie ausdrücken, oft ambig sind zwischen dem Ausdruck eines begrifflichen Zusammenhangs und der Aussage, dass eine bestimmte sprachliche Regel in Kraft ist. ${ }^{45}$ Im ersten Sinn sind die entsprechenden Sätze notwendig wahr, im zweiten Sinn kontingent. In dem Sinn, in welchem der Satz »Alle Erpel sind Erpel« zum Ausdruck bringt, dass eine bestimmte sprachliche Regel in Kraft ist, schließt er einen Fall aus. Aber nicht den Fall, dass die Welt derart ist, dass einige Erpel keine Erpel sind, sondern den Fall, dass wir andere sprachliche Regeln haben und die relevanten Ausdrücke so verwenden, dass die Regel »Alle Erpel sind Erpel« nicht in Kraft ist (z. B. weil »alle« etwas anderes bedeutet). In dem Sinn, in welchem der Satz »Alle Erpel sind Erpel« begrifflich wahr ist, schließt er aber, wie gesagt, keinen Fall aus und ist somit nicht-substanziell.

Es gibt auch noch einen zweiten Grund dafür, dass wir manchmal das Gefühl haben, dass begriffliche Wahrheiten einen Fall ausschließen, und zwar der, dass wir die begrifflichen Zusammenhänge nicht überschauen. Während wir auf den ersten Blick sehen, dass es keine Welt geben kann, in welcher einige Erpel keine Erpel sind, ist es weniger offensichtlich, dass es keine Welt

43 Vgl. Williamson 2007, S. 65.

44 Vgl. dazu auch Glock 2010, S. 344.

45 »Oft« und nicht »immer« deshalb, weil komplexe analytische Wahrheiten wie »Es ist möglich, dass der Vater meiner Halbschwester der Zwillingsbruder meiner Tante ist« nicht als sprachliche Regeln fungieren und deshalb nur die erste der beiden genannten Lesarten zulassen. 
geben kann, in welcher die Zeit rückwärts läuft. Aber trotzdem sind beide Aussagen begrifflich wahr und schließen mithin keinen Fall aus. Diese Art der Verwirrung wird sehr pointiert von John Wisdom zum Ausdruck gebracht:

The philosopher laments that we can never really know what is going on in someone else's mind, that we can never really know the causes of our sensations, that inductive conclusions are never really justified. He laments these things as if he can dream of another world where we can see our friends and tables face to face, where scientists can justify their conclusions and terriers can catch hares. ${ }^{46}$

Hier geht es aber natürlich nicht mehr um Frege-analytische Aussagen. Da sich aber auch in Bezug auf Letztere gezeigt hat, dass sie im relevanten Sinn nicht-substanziell sind, können wir contra Williamson folgern: Wären philosophische Wahrheiten im Allgemeinen Frege-analytisch, so könnte man mit Bezug auf diesen Umstand in der Tat einen philosophischen Exzeptionalismus begründen.

\subsection{Wahrheit aufgrund von Bedeutung?}

Im Gegensatz zu Freges Konzeption von Analytizität hält Williamson die Konzeption, analytische Wahrheiten seien wahr (allein) aufgrund ihrer Bedeutung nicht für brauchbar dafür, eine bestimmte Klasse von Sätzen herauszugreifen. Kein Satz, so Williamsons These, ist allein aufgrund seiner Bedeutung wahr. Deshalb ist das Kriterium der Wahrheit aufgrund von Bedeutung auch nicht dazu geeignet, analytische von synthetischen Urteilen abzugrenzen. Einer verbreiteten Idee zufolge, so Williamson, ist beispielsweise das synthetische Urteil »Alle Füchsinnen sind Omnivoren« wahr, (a) weil es bedeutet, dass alle Füchsinnen Omnivoren sind, und (b) weil tatsächlich alle Füchsinnen Omnivoren sind. Demgegenüber fällt bei analytischen Urteilen der Aspekt (b) weg: »Alle Füchsinnen sind Füchsinnen« ist demnach allein deshalb wahr, weil es bedeutet, dass alle Füchsinnen Füchsinnen sind. Dies stimmt aber Williamson zufolge nicht: Dass »Alle Füchsinnen sind Füchsinnen« bedeutet, dass alle Füchsinnen Füchsinnen sind, sei nur dann zur Begründung der Wahrheit dieses Urteils hinreichend, wenn schon vorausgesetzt werde, dass alle Füchsinnen Füchsinnen sind. Grundsätzlich, so Williamson, gilt für eine jede beliebige Wahrheit »p«, egal ob analytisch oder synthetisch: »p« ist wahr, weil es bedeutet, dass $p$, und weil $p .{ }^{47}$

\footnotetext{
$46 \quad$ Wisdom 1937, S. 104.

47 Vgl. Williamson 2007 , S. 58 f. Williamson beruft sich an der betreffenden Stelle auf Boghossian 1997, S. 335f. Der betreffende Punkt findet sich aber auch bereits in Lewis 1946 und Lewy 1976.
} 
An dieser Argumentation von Williamson lässt sich einiges kritisieren. Zunächst einmal sollte man weder im analytischen noch im synthetischen Fall sagen, ein Satz $» \mathrm{p}$ « bedeute, dass $p .{ }^{48}$ Die Formulierung $» x$ bedeutet, dass ...« hat eine Verwendung in Aussagen wie »Er ist krank, und das bedeutet, dass wir heute ohne ihn auskommen müssen « oder »diese Wolken bedeuten, dass es bald regnen wird «. In beiden Fällen besteht ein Zusammenhang in der Sache zwischen den Sachverhalten, auf den sich die Ausdrücke vor und nach »bedeutet« beziehen (`Er ist krank und `Wir müssen ohne ihn auskommen< respektive $>$ Diese Wolken $<$ und $>$ Es wird bald regnen $<$ ). Und $\gg x$ bedeutet, dass $p$ « ist faktiv: Daraus, dass etwas bedeutet, dass $p$, folgt, dass $p$. Wenn wir es demgegenüber mit sprachlicher Bedeutung zu tun haben, sollten wir der Deutlichkeit halber Formulierungen der folgenden Art wählen: »>Die Sonne scheint< bedeutet: Die Sonne scheint «, - oder gebräuchlicher: »The sun is shining 〈bedeutet: Die Sonne scheint«. Und diese Formulierung ist nicht faktiv: Natürlich folgt daraus, dass »The sun is shining « diese sprachliche Bedeutung hat, nicht, dass die Sonne scheint. ${ }^{49}$

Weiterhin buchstabiert Williamson die Auffassung, dass analytische Wahrheiten wahr aufgrund ihrer Bedeutung sind, aber auch auf eine völlig unplausible Art aus. Selbstverständlich ist »Junggesellen sind unverheiratet « nicht wahr, weil es bedeutet: Junggesellen sind unverheiratet. Sondern der Satz ist wahr aufgrund der Bedeutung der einzelnen Ausdrücke, aus denen er zusammengesetzt ist, und der Art ihrer Zusammensetzung. ${ }^{50}$ Und das ist es, was

48 Vgl. dazu auch Hacker 2009a, S. 344.

49 Zugegebenermaßen sind auch Formulierungen wie die folgende gebräuchlich: Dass eine Satz analytisch ist, bedeutet, dass er allein aufgrund seiner Bedeutung wahr ist. Auch diese Formulierung dient der Angabe sprachlicher Bedeutung, aber anders als in Williamsons Beispiel, das ich kritisiert habe, steht hier kein Ausdruck in Anführungszeichen vor »bedeutet«.

50 Auch diese Auffassung ist nicht ganz unproblematisch. Man kann zum Beispiel einwenden, die Wahrheit des analytischen Urteils »Junggesellen sind unverheiratete Männer« sei keine Konsequenz der Bedeutung von »Junggeselle« und anderen Wörtern. Vielmehr sei die Wahrheit dieses Satzes konstitutiv für die Bedeutung von »Junggeselle« (vgl. Baker/Hacker 2009, S. 314). »Junggeselle« bedeute also das, was es bedeute, aufgrund der Wahrheit von »Junggesellen sind unverheiratete Männer«, und nicht umgekehrt. Ich glaube jedoch (aus ähnlichen Gründen wie Glock 2009 und Schroeder 2009), dass sich diese beiden Relationen nicht ausschließen: »Junggesellen sind unverheiratete Männer« ist gleichzeitig eine Regel, die dem Wort »Junggeselle« seine Bedeutung gibt, und wahr aufgrund der sich aus dieser Regel ergebenden Bedeutung von »Junggeselle« und den anderen involvierten Wörtern. Und bei komplizierteren analytischen Wahrheiten wie der in Fußnote 45 genannten besteht ohnehin nur die eine der beiden Relationen: Solche Sätze sind wahr aufgrund der Bedeutung der in ihnen vorkommenden Wörter, aber sie sind ihrerseits keine Regeln für die Verwendung dieser Wörter. 
wir meinen, wenn wir sagen, eine Aussage sei wahr aufgrund ihrer Bedeutung. Dass die Wörter »Junggesellen«, »sind « und »unverheiratet« das bedeuten, was sie bedeuten, und in dieser Reihenfolge aneinandergefügt sind, ist bereits hinreichend dafür, dass das betreffende Urteil wahr ist: Es gibt nicht zusätzlich noch die Tatsache, dass Junggesellen unverheiratet sind. ${ }^{51}$ Denn wie bereits anhand von verschiedenen Beispielen ausgeführt, sind Urteile wie »Junggesellen sind unverheiratet" nicht-substanziell und ihre Wahrheit mithin davon unabhängig, was in der Welt der Fall ist.

Im Zusammenhang mit der Auffassung, synthetische Wahrheiten seien wahr aufgrund (a) ihrer Bedeutung und (b) dessen, was der Fall ist, während analytische Wahrheiten allein aufgrund von (a) wahr seien, spricht Williamson einen weiteren interessanten Punkt an. Und zwar behauptet er, es komme darin zu einer Äquivokation von »aufgrund ihrer Bedeutung«: im Falle von synthetischen Urteilen sei mit »Bedeutung« die Proposition gemeint, die eine einzelne Äußerung eines Satzes ausdrücke, während bei analytischen Urteilen die konventionelle Bedeutung eines bestimmten Satzes gemeint sei. ${ }^{52}$

Ich habe weiter oben bereits geschrieben, dass man die Wahrheit von »Junggesellen sind unverheiratet« nicht mit der konventionellen Bedeutung dieses Satzes begründen kann. Der Satz ist nicht deshalb wahr, weil er bedeutet: Junggesellen sind unverheiratet. Vielmehr ist er aufgrund der Bedeutung seiner Komponenten und der Art der Kombination derselben wahr. Bei synthetischen Urteilen, insbesondere bei indexikalischen, scheint es dagegen einfacher zu sein, den Bedeutungsaspekt über die von einer Äußerung zum Ausdruck gebrachten Aussage auszubuchstabieren, weshalb man prima facie geneigt sein könnte, Williamson darin recht zu geben, dass der oben genannte Aspekt (a) sich bei synthetischen Sätzen auf etwas anderes beläuft als bei analytischen. Es wäre relativ natürlich, die Wahrheit einer Äußerung von »Sie hat ihn gestern geheiratet« damit zu begründen, dass die Äußerung im betreffenden Kontext zum Ausdruck gebracht hat, dass Sarah Michael am 2. Februar 2017 geheiratet hat, und dass sich dies tatsächlich so zugetragen hat. Auf den zweiten Blick gibt es allerdings verschiedene Möglichkeiten, die Behauptung zu verteidigen, dass synthetische Wahrheiten aufgrund der oben genannten Faktoren (a) und (b) wahr sind und analytische Wahrheiten nur

51 Man könnte »Tatsache« natürlich auch deflationistisch auffassen, so dass gilt: Dass $p$, ist genau dann eine Tatsache, wenn » $p$ « wahr ist. Dann wäre es zwar eine Tatsache, dass Junggesellen unverheiratet sind, aber diese Tatsache wäre keiner der Gründe dafür, dass »Junggesellen sind unverheiratet« wahr ist: Der Satz wäre weiterhin allein aufgrund seiner Bedeutung wahr.

$5^{2}$ Vgl. Williamson 2007, S. 59, Fußnote 10. 
aufgrund von (a), und (a) so auszubuchstabieren, dass es im Falle beider Arten von Wahrheiten auf dasselbe hinausläuft:

\section{Möglichkeit 1: In beiden Fällen Äußerungsbedeutung}

Hacker zufolge haben wir es auch bei analytischen Wahrheiten nicht mit der Wahrheit von Sätzen, sondern mit derWahrheit einzelner Äußerungen oder Verwendungen von Sätzen zu tun. Dieser Punkt ist ihm besonders deshalb wichtig, weil je nach Kontext ein und derselbe Satz eine synthetische Aussage oder eine analytische Wahrheit ausdrücken kann. ${ }^{53}$ Ein oft zitiertes Beispiel dafür ist »Krieg ist Krieg«. Dieser Satz kann als Beispiel für eine analytische Wahrheit gebraucht werden, kann aber in anderen Kontexten auch etwa zum Ausdruck bringen, dass alle Kriege gleich sind oder dass im Krieg andere Regeln gelten als außerhalb des Krieges. Und auch in den Wissenschaften kann ein Satz in einem Kontext eine synthetische Wahrheit ausdrücken und in einem anderen Kontext eine analytische Wahrheit, weil sich die Konventionen verändern können und beispielsweise eine einstige Beobachtung in die Definition eines Wortes eingehen kann. So dürfte es auch mit »Wasser « und $» \mathrm{H}_{2} \mathrm{O}$ « passiert sein: »Wasser ist $\mathrm{H}_{2} \mathrm{O}$ « drückte zunächst eine wissenschaftliche Entdeckung aus und später eine begriffliche Wahrheit.

\section{Möglichkeit 2: Verschiedene Arten von Bedeutungsträgern}

Bei klassischen Beispielen analytischer Wahrheiten wie »Junggesellen sind unverheiratet« oder »Erpel sind männliche Enten « spricht allerdings meines Erachtens nicht viel dagegen, zu behaupten, dass die betreffenden Sätze analytisch sind: Auch wenn man sich vielleicht abenteuerliche Kontexte ausmalen kann, in welchen diese Sätze zur Äußerung eines synthetischen Urteils verwendet werden könnten, kann man zumindest sagen, dass sie in ihrer Standard-Bedeutung analytisch sind. Und diese Redeweise scheint mir auch besser als die Möglichkeit 1 der Weise Rechnung zu tragen, in der wir normalerweise über analytische Wahrheiten sprechen: Wir sagen, dass »Junggesellen sind unverheiratet « analytisch ist, und schränken diese Aussage nicht auf einzelne Äußerungen oder Verwendungen dieses Satzes ein.

Natürlich gibt es auch Ausdrücke mit mehreren Standardbedeutungen. »Bank « ist zum Beispiel ambig, und deshalb ist auch »Jede Bank ist ein Finanzinstitut« in der sich durch die konventionelle Bedeutung der Satzkomponenten ergebenden Standard-Bedeutung ambig zwischen einer analytischen Wahrheit und einer (begrifflichen, nicht synthetischen) Unwahrheit. In derselben Weise sind auch Sätze denkbar, die eine analytische und eine synthetische 
Standard-Bedeutung haben, ${ }^{54}$ was dafür sprechen würde, wie von Hacker vorgeschlagen, von analytischen Satz-Verwendungen statt von analytischen Sätzen zu sprechen. Aber natürlich können wir auch bei »Jede Bank ist ein Finanzinstitut « sagen, dass der Satz - in einer seiner Standard-Bedeutungen analytisch ist. Und ebenso bei den antizipierten Beispielen von Sätzen, die sowohl analytische als auch synthetische Urteile ausdrücken können. Ich sehe jedenfalls kein Totschlagargument gegen die Rede von analytischen Sätzen. Bei synthetischen Urteilen ist es demgegenüber eindeutiger, dass es die Äußerungen, nicht die Sätze sind, die wahr oder falsch sind (obschon diese Unterscheidung auch dort bei kontextunabhängigen Urteilen wie »Die Erde dreht sich um die Sonne « nicht besonders relevant ist).

Wenn wir nun aber sagen: Synthetische Wahrheiten sind Äußerungen, analytische Wahrheiten sind Sätze, dann benötigen wir gar keine Äquivokation von »aufgrund ihrer Bedeutung«, damit dieser Aspekt in den beiden Fällen auf Unterschiedliches hinausläuft. Das von Williamson diskutierte Prinzip könnte dann auch wie folgt formuliert werden:

Für die Wahrheit oder Falschheit der Äußerungen synthetischer Sätze sind zwei Dinge relevant: Ihre Bedeutung und was der Fall ist. Für die Wahrheit (oder Falschheit ${ }^{55}$ ) analytischer Sätze ist demgegenüber einzig ihre Bedeutung relevant.

Und die Bedeutung einer Äußerung ist nun einmal etwas anderes als die Bedeutung eines Satzes, auch ohne Ambiguität von »Bedeutung«.

\section{Möglichkeit 3: Character vs. Content}

Wen die ersten beiden Möglichkeiten zur Rettung der Behauptung, synthetische Wahrheiten seien aufgrund der oben genannten Aspekte (a) und (b) wahr und analytische Wahrheiten nur aufgrund von (a), nicht zufriedenstellen, dem bleibt noch eine dritte Alternative. Und zwar könnte man (a) sowohl im Falle von synthetischen als auch im Falle von analytischen Wahrheiten mit den konventionellen Satzbedeutungen identifizieren: Man kann die Bedeutung von Sätzen wie »Sie hat ihn gestern geheiratet« auch angeben, ohne zu bestimmen,

54 Man könnte sich darüber streiten, ob man in den betreffenden Fällen von einem Wort bzw. Satz mit zwei Bedeutungen oder von zwei verschiedenen, gleichlautenden Wörtern bzw. Sätzen sprechen sollte. Ich tendiere eher zur ersten Formulierung, aber Schroeder präferiert beispielsweise die zweite (vgl. Schroeder 2009, S. 93f., insb. Fußnote 26).

55 Manche Philosophinnen und Philosophen sprechen sowohl von »analytisch wahren « als auch von »analytisch falschen« Sätzen, andere schränken das Prädikat »analytisch« auf begriffliche Wahrheiten ein. Ich schließe mich, wo nicht anders angegeben, der zweiten Verwendungsweise an. 
wer »sie« und »er« sind und auf welches Datum sich »gestern« bezieht. Die Angabe der Bedeutung schließt für diese indexikalischen Ausdrücke die Regeln zur Festlegung davon ein, worauf sich die Ausdrücke in einzelnen Äußerungskontexten beziehen - das, was David Kaplan »Character« nennt. Der Content oder die durch die spezifische Äußerung zum Ausdruck gebrachte Aussage wird dann zusammen mit der Wahrheit oder Falschheit durch (b) festgelegt: durch das, was der Fall ist. Der Aspekt (b) ist dieser Konzeption zufolge also in zweifacher Weise relevant für die Wahrheit eines synthetischen Urteils: Bestimmte Tatsachen sind dafür ausschlaggebend, dass sich »sie«, »ihn« und »gestern« auf Sarah, Michael und den 2. Februar 2017 beziehen. Andere Tatsachen sind dafür ausschlaggebend, dass die sich auf dieser Grundlage ergebende, spezifische Aussage wahr ist. Auf der (a)-Seite bleibt nur die konventionelle Bedeutung des Satzes übrig. ${ }^{56}$

Williamsons Behauptung, dass wir es mit einer Äquivokation von »aufgrund ihrer Bedeutung« zu tun haben, wenn wir diesen Aspekt sowohl bei synthetischen als auch bei analytischen Wahrheiten als einen der beiden Faktoren identifizieren, die für ihre Wahrheit ausschlaggebend sind, kann angesichts der verschiedenen beschriebenen Argumentationsmöglichkeiten angezweifelt werden.

Unabhängig von Williamsons Argumentation ist es aber ohnehin fraglich, ob wir über synthetische Wahrheiten sagen wollen, dass sie wahr sind einerseits aufgrund ihrer Bedeutung und andererseits aufgrund dessen, was der Fall ist. Denn wenn wir uns fragen, ob eine Äußerung einer synthetischen Aussage wahr oder falsch ist bzw. weshalb sie wahr oder falsch ist, setzen wir die Bedeutung der Äußerung üblicherweise voraus. Aufgrund welches Umstands ist Peters Äußerung von »Es stehen vier Flaschen auf dem Tisch« wahr? Die Antwort ist: Aufgrund der Tatsache, dass vier Flaschen auf dem Tisch stehen. Die Bedeutung einer synthetischen Aussage gibt man normalerweise nur

56 Man könnte gegen die in diesem Absatz ausgeführte Idee einwenden, dass es auch analytische Sätze gibt, die indexikalische Ausdrücke enthalten. Wenn die Festlegung der Bezugsobjekte dieser Ausdrücke zum Aspekt (b) gehört, müsste in die Begründung der Wahrheit dieser Sätze dann auch einfließen, was der Fall ist, und dies ist nicht mit der zur Diskussion stehenden Definition analytischer Wahrheiten kompatibel. Aber erstens kommen Sätze wie »Dieser Tisch ist ein Tisch « (geäußert, während auf ein Objekt gezeigt wird) ohnehin nur dann als analytische Wahrheiten in Frage, wenn irrelevant ist, worauf bei der Äußerung gezeigt wird (und ob überhaupt auf etwas gezeigt wird); sonst könnten sie nämlich auch unwahr oder ohne Wahrheitswert (und somit nicht analytisch wahr) sein. Der Aspekt (b) spielt zur Begründung ihrer Wahrheit also so oder so keine Rolle. Und zweitens könnte in Frage gestellt werden, ob wir solche Sätze überhaupt als sinnvoll auffassen sollten, da es sich bei ihnen um seltsame Hybriden von begrifflichen Wahrheiten und Urteilen über einen bestimmten Gegenstand handelt. 
dann als zusätzlichen Faktor an, der für ihre Wahrheit relevant ist, wenn wir es mit einem fremdsprachigen Satz zu tun haben oder mit einem Satz, der erläuterungsbedürftige Ausdrücke enthält: Wieso ist $» B$ нашей солнечной системе восемь планет « wahr? Weil es bedeutet: In unserem Sonnensystem gibt es acht Planeten. Weil Planeten so-und-so definiert sind. Und weil es in unserem Sonnensystem acht Objekte gibt, die diese Definition erfüllen.

Natürlich stimmt es, dass »Es stehen vier Flaschen auf dem Tisch« nicht nur dann unwahr wäre, wenn nicht tatsächlich vier Flaschen auf dem Tisch stehen würden, sondern auch beispielsweise dann, wenn »Flaschen« Stühle bedeuten würde. Trotzdem ist der Umstand, dass »Es stehen vier Flaschen auf dem Tisch « das bedeutet, was es bedeutet, nicht etwas, das wir normalerweise als einen der Gründe für die Wahrheit einer entsprechenden Äußerung anführen würden, sondern etwas, das wir bei der Begründung der Wahrheit voraussetzen.

Bei analytischen Sätzen verhält es sich anders. Dort gibt es keine Tatsachen, die für die Wahrheit entsprechender Äußerungen relevant sind, ${ }^{57}$ und der Umstand, dass jemand die Frage stellt, weshalb ein solcher Satz wahr ist, deutet bereits darauf hin, dass diese Person sich entweder nicht bewusst ist, dass für die Wahrheit bestimmter Sätze keine Tatsachen relevant sind, oder dass sie die Bedeutung der Äußerung eben nicht voraussetzt. Deshalb ist es bei der Beantwortung der Frage, weshalb beispielsweise »Alle Junggesellen sind unverheiratet« wahr ist, weniger inadäquat als bei synthetischen Urteilen zu sagen, der Satz sei wahr aufgrund seiner Bedeutung. Anstelle der Konzeption, synthetische Wahrheiten seien wahr aufgrund (a) ihrer Bedeutung und (b) dessen, was der Fall ist, während analytische Wahrheiten allein aufgrund von (a) wahr seien, würde man deshalb besser sagen:

Synthetische Wahrheiten sind wahr aufgrund von Tatsachen, analytische Wahrheiten sind wahr aufgrund ihrer Bedeutung.

\section{Epistemologische Konzeptionen von Analytizität}

\subsection{Definition epistemologischer Analytizität}

Wie oben besprochen, geben uns Williamson zufolge metaphysische Konzeptionen von Analytizität kein Mittel dafür an die Hand, einen philosophischen Exzeptionalismus zu begründen. Epistemologische Konzeptionen

57 Dies gilt natürlich nur, wenn wir die analytischen Aussagen nicht als Urteile darüber lesen, dass eine bestimmte Regel in Kraft sei. Vgl. weiter oben, S. 148. 
von Analytizität sind in dieser Hinsicht vielversprechender, so Williamson, da wir nach diesen Konzeptionen per Definition einen besonderen Zugang zu analytischen Urteilen zu haben scheinen. Eine Möglichkeit, analytische Wahrheiten epistemologisch zu definieren, ist die folgende:

Ein Satz ist genau dann analytisch, wenn es nicht möglich ist, ihn zu verstehen, ohne ihm zuzustimmen.

Die sich aus dieser Definition ergebende Konzeption analytischer Wahrheit steht im Zentrum von Williamsons Besprechung des Themas Analytizität. Williamson lehnt diese Auffassung mit der Begründung ab, dass es keine Sätze gibt, denen man notwendigerweise zustimmt, wenn man sie versteht. Außerdem macht er wie bereits oben bei den metaphysischen Konzeptionen geltend, dass aus dieser Konzeption analytischer Wahrheiten - zusammen mit der Idee, philosophische Kernwahrheiten seien in diesem Sinn analytisch nicht automatisch ein methodologischer Exzeptionalismus der Philosophie folge. Denn dass ein Satz im oben bestimmten Sinn analytisch ist, lässt nicht unmittelbar Rückschlüsse darauf zu, worauf unser Wissen, dass er wahr ist, gründet: Die in der Definition beschriebenen Verstehens-ZustimmungsVerbindungen (understanding-assent links) gehen nicht zwingend einher mit entsprechenden Verstehens-Wissens-Verbindungen.

Beide Argumente von Williamson, sowohl die Behauptung, es gebe keinen notwendigen Zusammenhang zwischen dem Verstehen bestimmter Sätze und der Zustimmung zu ihnen, als auch das Problem, dass sich ihm zufolge beim Übergang von Verstehens-Zustimmungs-Verbindungen zu VerstehensWissens-Verbindungen ergibt, sind auch für solche Philosophinnen und Philosophen relevant, die analytische Wahrheiten (wie ich selbst) semantisch (oder metaphysisch) definieren, aber zusätzlich die Auffassung vertreten, dass das Verstehen analytischer Sätze (wenigstens in vielen Fällen) hinreichend dafür ist, zu wissen, dass sie wahr sind.

\subsection{Zustimmung zu unwahren Urteilen}

Woher wissen wir, dass alle Junggesellen männlich sind? Einige Philosophinnen und Philosophen sind der Auffassung, dass wir dieses Wissen zusammen mit unserer Sprachkompetenz erlangen. Gehen wir für den Moment davon aus, dass es zwischen dem Verstehen des Satzes »Alle Junggesellen sind männlich « und der Überzeugung, dass dieser Satz wahr ist, einen notwendigen Zusammenhang gibt. Damit wir sagen können, dass Personen, die den Satz verstehen, auch wissen, dass er wahr ist, benötigen wir nach herkömmlicher Ansicht zusätzlich zur entsprechenden Überzeugung mindestens auch (a) die 
Wahrheit der Überzeugung und (b) eine Rechtfertigung für die Überzeugung. Man könnte argumentieren: In Fällen wie »Alle Junggesellen sind männlich«, in welchen es Verstehens-Zustimmungs-Verbindungen gibt, legt die etablierte Verwendung der in die betreffenden Sätze involvierten Wörter fest, dass diese Sätze wahr sind. Und das Verstehen der Sätze beziehungsweise die Vertrautheit mit den etablierten Regeln zur Verwendung ihrer Teilausdrücke stattet einen nicht nur mit der Überzeugung aus, dass die Sätze wahr sind, sondern auch mit einer entsprechenden Rechtfertigung.

Was hindert uns dann aber daran, fragt Williamson, dasselbe auch in Bezug auf »Phlogiston « und das Urteil »Alle brennbaren Körper enthalten Phlogiston « zu behaupten? Das Verstehen dieses Satzes dürfte, wenigstens im 17. Jahrhundert, in demselben Verhältnis zur Überzeugung, dass er wahr ist, gestanden haben, wie das Verstehen von »Alle Junggesellen sind männlich« heute zur Zustimmung zu diesem Satz steht, meint Williamson. Zwingt uns die Argumentation aus dem vorangehenden Absatz dann nicht dazu, zu sagen, dass die Leute im 17. Jahrhundert wussten, dass alle brennbaren Körper Phlogiston enthielten? Dies wäre natürlich eine absurde Konsequenz, da wir heute wissen, dass es Phlogiston nicht gibt.

Analog könnte man auch im Zusammenhang mit Arthur Priors »tonk« und dem Urteil, dass aus jedem Urteil Beliebiges folgt, argumentieren, ${ }^{58}$ und unter Umständen auch im Zusammenhang mit abwertenden Ausdrücken wie »Schlitzauge« oder »Kanake« und den Urteilen, dass Ostasiaten respektive Südländer verachtenswert sind. ${ }^{59}$

Ein weiteres, etwas komplexeres Beispiel, das Williamson anspricht, ist das Wort »wahr«. Es wird manchmal behauptet, dass es eine notwendige Bedingung für das Verstehen von »wahr« ist, dass man dem folgenden Satz zustimmt:

(10) Für jede beliebige Aussage $» \mathrm{p}$ « gilt: $» \mathrm{p}$ « ist genau dann wahr, wenn $p$.

Diese Regel gibt allerdings Anlass zum Lügner-Paradox. Wollen wir trotzdem sagen, dass (10) wahr ist und dass kompetente Sprecherinnen und Sprecher wissen, dass »p « genau dann wahr ist, wenn $p ?^{60}$

Bei manchen dieser Beispiele ergibt sich das Problem in Wirklichkeit überhaupt nicht beim Übergang von einer Verstehens-Zustimmungs-Verbindung zu einer Verstehens-Wissens-Verbindung, da gar keine entsprechende VerstehensZustimmungs-Verbindung besteht: Es ist möglich, »Phlogiston«, »Schlitzauge«

\footnotetext{
$58 \quad$ Vgl. Williamson 2007, S. 79f., Prior 1960.

59 Vgl. Williamson 2007, S. 80, Fußnote 4. Williamson äußert sich allerdings selbst skeptisch in Bezug darauf, solche abwertenden Ausdrücke als einschlägige Beispiele aufzufassen.

6o Vgl. Williamson 2007, S. 79 .
} 
oder »Kanake« zu verstehen, ohne den Urteilen zuzustimmen, dass alle brennbaren Körper Phlogiston enthalten oder dass Ostasiaten oder Südländer verachtenswert sind. Und für »tonk « gilt unter Umständen dasselbe; man könnte stattdessen aber auch mit Williamson dafür argumentieren, das »tonk«Beispiel deshalb außer Acht zu lassen, weil es in diesem Fall gar nichts zu verstehen gibt. ${ }^{61}$

War es vielleicht früher unmöglich, »Phlogiston« (und »brennbar«, »Körper« usw.) zu verstehen, ohne dem Urteil »Alle brennbaren Körper enthalten Phlogiston « zuzustimmen? Selbst wenn dieser Ausdruck ursprünglich einmal mit den Worten »Phlogiston ist die Substanz, die alle brennbaren Körper enthalten und aufgrund derer diese Körper brennbar sind « eingeführt worden wäre - also unter Rückgriff auf eine definite Kennzeichnung, die die Existenz einer entsprechenden Substanz präsupponiert - ist anzunehmen, dass sich die Verwendung von »Phlogiston« dann so etabliert hätte, dass »Phlogiston existiert nicht« auch bereits im 17. Jahrhundert eine verständliche Aussage gewesen wäre, und keine Kontradiktion. Und wenn »Phlogiston existiert nicht« damals keine verständliche Aussage gewesen wäre, hätte es notwendigerweise etwas anderes bedeutet als das, was später bewiesen wurde: dass es keine Substanz gibt, die in allen brennbaren Körper enthalten ist und für ihre Brennbarkeit verantwortlich ist. Denn kein Urteil kann gleichzeitig begrifflich notwendig oder inkonsistent und empirisch wahr oder falsch sein. Es ist also unmöglich, eine empirische Wahrheit durch die Einführung eines neuen Begriffs zu einer begrifflichen Wahrheit zu machen: Wenn wir mit der Einführung bestimmter Begriffe beispielsweise stipulieren, dass die Sätze »Gold ist schwerer als Blei« oder »Es gibt Platin« neuerdings begrifflich wahr sind, dann haben wir eo ipso die Bedeutung dieser Sätze verändert und die Sätze bringen in ihren neuen Bedeutungen keine empirischen Urteile mehr zum Ausdruck.

Wie gesagt, besteht aber das realistischere Szenario darin, dass »Alle brennbaren Körper enthalten Phlogiston « auch bereits im 17. Jahrhundert ein empirisches Urteil ausgedrückt hat, dessen Wahrheit man bestreiten konnte, ohne damit einen begrifflichen Fehler zu begehen. Es war also immer möglich, das Wort »Phlogiston « und die anderen relevanten Wörter zu verstehen und trotzdem dem Satz »Alle brennbaren Körper enthalten Phlogiston« zu widersprechen.

Wie ist es mit »wahr « und dem oben genannten Satz (10)? Auch hier ist nicht ohne Weiteres klar, dass eine entsprechende Verstehens-Zustimmungs-

Vgl. Williamson 2007, S. 81. 
Verbindung besteht. Da (10) wesentlich komplexer ist als beispielsweise »Alle Junggesellen sind männlich«, ist es im Falle von (10) zumindest einfacher als im anderen Fall zu behaupten, man könne sämtliche Komponenten des Satzes verstehen und sich auch seiner Konstruktionsweise bewusst sein, ohne der Überzeugung zu sein, dass er wahr ist. Allerdings können wir andere Fälle konstruieren, in welchen kein solcher Ausweg besteht: Stellen wir uns eine Sprachgemeinschaft vor, die sich von der unsrigen dadurch unterscheidet, dass Kurt Grellings Term »heterologisch« dort gebräuchlich ist. Dieses Wort ist wie folgt definiert:

(11) Ein Adjektiv ist genau dann heterologisch, wenn es nicht auf sich selbst zutrifft.

Aus irgendeinem Grund ist es den Mitgliedern der betreffenden Sprachgemeinschaft sehr wichtig, ob Adjektive heterologisch sind oder nicht, deshalb verwenden sie den Ausdruck häufig. Und dabei haben sie immer die Verwendungsregel (11) im Sinn. In diesem Fall scheint für den Satz (11) in der Tat eine Verstehens-Zustimmungs-Verbindung zu bestehen. Aber es ist problematisch, zu sagen, dass (11) wahr sei, da dieser Satz bekanntlich zu einem Paradox Anlass gibt: Ist das Adjektiv »heterologisch« selbst heterologisch oder nicht heterologisch? Entweder wir sagen, es sei heterologisch. Heterologisch zu sein, bedeutet aber, nicht auf sich selbst zuzutreffen. Und wenn »heterologisch« nicht auf »heterologisch« zutrifft, müsste heterologisch entgegen unserer Annahme nicht heterologisch sein. Oder wir sagen, »heterologisch « sei nicht heterologisch. Dann trifft es nicht auf sich selbst zu, was aber wiederum implizieren würde, dass es heterologisch ist. Haben wir es hier also mit einer Verstehens-Zustimmungs-Verbindung zu tun, die nicht mit einer Verstehens-Wahrheits-Verbindung einhergeht? Nicht wirklich. In einem gewissen Sinn ist der Satz (15) im beschriebenen Szenario nämlich tatsächlich wahr: Dieser Regel wird in der zur Diskussion stehenden Sprachgemeinschaft tatsächlich gefolgt, und der Satz (15) ist die korrekte Definition des Wortes $»$ heterologisch $\ll$.

Wenn wir trotzdem darauf bestehen wollen, dass (15) nicht wahr sei, da unmöglich alle und nur diejenigen Adjektive heterologisch sein können, die nicht auf sich selbst zutreffen, scheint es mir, dass wir die zu Beginn des vorliegenden Abschnitts dargelegte Auffassung über den Zusammenhang zwischen Sprachkompetenz und Wissen dennoch nicht aufgeben müssen. Wir können dann einfach als Ausnahme hinzufügen, dass die Kenntnis einer sprachlichen Regel nicht zu Wissen über Begriffszusammenhänge führt, wenn die betreffende Regel inkonsistent ist. 


\subsection{Verstehen ohne Zustimmung}

4.3.1 Peter und Stephen

Wichtiger (und in der Literatur auch viel häufiger diskutiert) als das im vorangehenden Abschnitt besprochene Argument von Williamson ist sein Argument dafür, dass es möglich ist, den Satz »Alle Füchsinnen sind Füchsinnen« zu verstehen, ohne diesem Satz zuzustimmen. Williamson beruft sich in seiner diesbezüglichen Argumentation auf zwei fiktive Philosophen: Peter und Stephen.

Peter glaubt, dass es keine Füchsinnen gibt und dass Sätze der Form »Alle $F$ sind $G$ « nur dann wahr sein können, wenn mindestens ein $F$ existiert. Deshalb stimmt er dem Satz »Alle Füchsinnen sind Füchsinnen« nicht zu. Stephen ist Vertreter einer dreiwertigen Logik, in der es neben »wahr« und »falsch« auch noch den Wahrheitswert »unbestimmt« gibt. Bei Grenzfällen für das Fallen unter einen Begriff hat das betreffende Urteil »x ist $F \ll$ den Wahrheitswert »unbestimmt«. Auch Sätze der Form $» x$ ist $F \rightarrow x$ ist $G$ « haben Stephen zufolge den Wahrheitswert »unbestimmt«, wenn sowohl das Antezedens als auch das Konsequens diesen Wahrheitswert hat. Weiterhin ist Stephen der Auffassung, dass es Grenzfälle für das Fallen unter den Begriff Füchsin gibt und dass mithin einige Instanzen von $» x$ ist eine Füchsin $\rightarrow x$ ist eine Füchsin « unbestimmt sind. Auch das Urteil »Alle Füchsinnen sind Füchsinnen« ist deshalb Stephen zufolge nicht wahr, sondern unbestimmt, und er stimmt diesem Urteil mithin nicht zu.

Williamson führt Peter und Stephen als mögliche Beispiele für Personen an, die den Satz »Alle Füchsinnen sind Füchsinnen « verstehen, ohne ihm zuzustimmen. Aber sollten wir tatsächlich sagen, dass Peter und Stephen den Satz »Alle Füchsinnen sind Füchsinnen« verstehen, wenn sie andere Anforderungen an die Wahrheit des Satzes stellen als gewöhnliche kompetente Sprecherinnen und Sprecher?62 Und sollten wir nicht wenigstens sagen, dass sie den Satz anders verstehen als wir und die Verstehens-ZustimmungsVerbindung so eingrenzen, dass sie bedeutet: Man kann den Satz nicht in seiner gewöhnlichen Bedeutung verstehen, ohne ihm zuzustimmen? Williamson listet eine ganze Reihe von Aspekten auf, die ihm zufolge dafür sprechen, zu sagen,

62 Im Grunde genommen ist es gar nicht so klar, ob Peters Verständnis von »Alle Füchsinnen sind Füchsinnen « von der gewöhnlichen Bedeutung dieses Satzes abweicht. Dass dieses Urteil die Existenz von mindestens einer Füchsin präsupponiert, könnte durchaus einer der etablierten Auffassungen dieses Satzes entsprechen. Dieser Umstand ist für die vorliegende Diskussion aber nicht relevant, da man sich auch Sprecherinnen und Sprecher ausdenken könnte, die aus irgendwelchen seltsamen Gründen einer beliebigen anderen analytischen Wahrheit widersprechen würden. Stephen, im Gegensatz zu Peter, würde zum Beispiel auch dem Satz »Wenn etwas eine Füchsin ist, ist es eine Füchsin« nicht zustimmen. 
dass Peter und Stephen den Satz »Alle Füchsinnen sind Füchsinnen« verstehen und dass sie ihn in seiner gewöhnlichen Bedeutung verstehen:

a) Im Gespräch mit Peter und Stephen hat man normalerweise den Eindruck, dass sie die Wörter »Alle«, »Füchsin« und die anderen im oben genannten Satz vorkommenden Wörter verstehen. ${ }^{63}$

b) Wir können uns sogar vorstellen, dass Peter und Stephen philosophische Artikel zu den Wahrheitsbedingungen von Allaussagen publiziert hätten. Die Leserinnen und Leser dieser Artikel würden wohl kaum die Sprachkompetenz von Peter und Stephen in Zweifel ziehen, sondern eher zur Auffassung gelangen, dass die beiden seltsame Ansichten (»views«) vertreten. ${ }^{64}$

c) Peter und Stephen haben nicht die Absicht, den Satz »Alle Füchsinnen sind Füchsinnen « in einer Bedeutung zu verwenden, die von der gewöhnlichen Bedeutung abweicht, die der Satz im Deutschen hat. Wenn man ihnen beweisen könnte, dass ihre Theorien über die Wahrheitsbedingungen von Allaussagen nicht korrekt sind für das Deutsche, wie es von der Allgemeinheit gesprochen wird, würden sie die Theorien sofort aufgeben. ${ }^{65}$

d) Dass man eine falsche Theorie über die Bedeutung eines Ausdrucks vertritt, bedeutet nicht, dass man den Ausdruck tatsächlich falsch beziehungsweise von der Norm abweichend verwendet. ${ }^{66}$

e) Dass Peter und Stephen dem Satz »Alle Füchsinnen sind Füchsinnen« nicht zustimmen, mag prima facie Zweifel daran wecken, dass sie den Satz richtig verstehen. Aber diese Zweifel werden durch den Umstand beseitigt, dass sie sämtliche im Satz vorkommenden Wörter in anderen Kontexten völlig normal verwenden. ${ }^{67}$

f) Peter und Stephen sind gewöhnliche kompetente Sprecher, die von klein auf Deutsch gesprochen haben. In ihrer Kindheit und Jugend hätten sie den Satz »Alle Füchsinnen sind Füchsinnen« sofort als wahr akzeptiert. Sie sind erst später aufgrund bestimmter Überlegungen zum Schluss gelangt, dass der Satz in Wirklichkeit nicht wahr ist. Aber es gibt überhaupt keinen Grund, anzunehmen, dass sie zu irgendeinem Zeitpunkt vergessen haben, was der Satz »Alle Füchsinnen sind Füchsinnen« bedeutet. ${ }^{68}$

63 Vgl. Williamson 2007 , S. 88 f.

64 Vgl. Williamson 2007, S. 89.

65 Vgl. Williamson 2007 , S. 89.

66 Vgl. Williamson 2007, S. 89.

67 Vgl. Williamson 2007 , S. 97.

68 Vgl. Williamson 2007, S. 90. 
g) Wenn jemand sprachliche Ausdrücke nicht richtig versteht, bietet es sich in der Regel an, dieser Person die Bedeutung dieser Ausdrücke zu erklären. Peter und Stephen befinden sich aber offensichtlich nicht in einer Situation, in der es angemessen wäre, ihnen die Bedeutung von »Alle Füchsinnen sind Füchsinnen« (oder von einer der Komponenten dieses Satzes) zu erklären. ${ }^{69}$

h) Peters und Stephens Auffassung von »Alle Füchsinnen sind Füchsinnen« weicht nicht semantisch, sondern logisch von unserer Auffassung ab. ${ }^{70}$

Einige dieser Aspekte sind als Argumente dafür, dass Peter und Stephen den zur Diskussion stehenden Satz im gewöhnlichen Sinn verstehen, vollkommen wertlos. Dass normalerweise im Gespräch mit Peter und Stephen nicht der Eindruck entsteht, dass sie »alle « (und die anderen in den Satz involvierten Wörter) falsch verstehen (a), ist vereinbar damit, dass sie dieses Wort in einem leicht abweichenden Sinn verstehen. Auch der Aspekt (f) passt zu dieser Hypothese: Dass Peter und Stephen den Satz »Alle Füchsinnen sind Füchsinnen « in einem Sinn verstehen, der nur leicht vom gewöhnlichen Sinn des Satzes abweicht, wird nur in wenigen Kontexten zum Tragen kommen. Dass Peter und Stephen die zur Diskussion stehenden Ausdrücke im gewöhnlichen Sinn zu verwenden beabsichtigen (c), schließt natürlich nicht aus, dass sie die Ausdrücke falsch verstehen. Und dass sie unter kontrafaktischen Bedingungen ihre Auffassung der Bedeutung von "Alle Füchsinnen sind Füchsinnen" revidieren würden (ebenfalls (c)), ist damit kompatibel, dass sie gegenwärtig eine abweichende Auffassung davon haben.

Dass Peter und Stephen früher das Wort »alle« richtig verstanden haben und jetzt nicht mehr richtig verstehen, impliziert außerdem nicht, dass sie die Bedeutung dieses Wortes vergessen haben (f). Zum Vergleich: Über jemanden, der von einem Verschwörungstheoretiker davon überzeugt worden ist, dass es sich bei den weißen Streifen am Himmel um Chemikalien handelt, die von der amerikanischen Luftwaffe zur Vergiftung der Bevölkerung versprüht werden, müssen wir auch nicht sagen, er habe vergessen, dass diese Streifen harmlose, kondensierte Flugzeugabgase sind. Dennoch findet auch in diesem Beispiel ein Übergang von Wissen zu Nicht-Wissen statt.

Und schließlich: Dass es unangemessen wäre, Peter und Stephen zu erklären, was »alle« beziehungsweise »Alle Füchsinnen sind Füchsinnen « bedeutet (g), liegt an der besonderen Weise, in der ihr Missverstehen des Satzes zustande gekommen ist und spricht nicht dagegen, dass sie diese Ausdrücke falsch verstehen. Wenn ein Kind oder eine Sprecherin einer anderen Sprache einen

69 Vgl. Williamson 2007, S. 91.

70 Vgl. Williamson 2007, S. 91, 115 . 
Ausdruck falsch versteht, sind ganz einfach andere Maßnahmen angebracht als wenn eine Philosophin oder ein Philosoph aufgrund einer theoretischen Überlegung über die Bedeutung eines Ausdrucks in Verwirrung gerät.

$\mathrm{Zu}$ den anderen angeführten Aspekten ist ein bisschen mehr zu sagen:

$\mathrm{Zu}(\mathrm{b})$ : Dass Peter und Stephen den Satz »Alle Füchsinnen sind Füchsinnen « in einem Sinn verstehen, der leicht vom gewöhnlichen Sinn des Satzes abweicht, ist problemlos mit dem Umstand vereinbar, dass die beiden im Allgemeinen sprachkompetent sind. Um ein kompetenter Sprecher oder eine kompetente Sprecherin einer Sprache zu sein, muss man nicht alle Wörter dieser Sprache verstehen und richtig verwenden. Und wenn Peter und Stephen in ihren Artikeln schreiben, der Satz »Alle Füchsinnen sind Füchsinnen« sei nicht wahr, wenn es keine Füchsinnen gebe (Peter) beziehungsweise wenn es Grenzfälle für das Fallen unter »Füchsin« gebe (Stephen), dann kann es natürlich sein, dass die Leserinnen und Leser sagen würden, Peter und Stephen hätten seltsame Ansichten über die Wahrheitsbedingungen von »Alle Füchsinnen sind Füchsinnen «, ${ }^{71}$ und nicht, Peter und Stephen hätten seltsame Auffassungen der Bedeutung dieses Satzes. Aber tatsächlich impliziert die erste Behauptung die zweite: Einem Satz von der Norm abweichende Wahrheitsbedingungen zuzuschreiben, bedeutet, ihm eine von der Norm abweichende Bedeutung zuzuschreiben. Denn zwei Sätze können nicht dieselbe Bedeutung, aber unterschiedliche Wahrheitsbedingungen haben.

$\mathrm{Zu}$ (d): Es stimmt, dass man einen Ausdruck auch dann korrekt verwenden kann, wenn man sich auf theoretischer Ebene darüber irrt, wie er verwendet wird. Wir können uns zum Beispiel, wie bereits in Kapitel 2 erwähnt, einen Sprecher vorstellen, der das Prädikat »x lügt« vollkommen richtig verwendet, aber auf Anfrage behaupten würde, es finde genau dann auf eine Person Anwendung, wenn diese Person etwas Unwahres sage. Da dieser Sprecher Fälle, in welchen ein Subjekt $S$ etwas sagt, das $S$ für wahr hält, obwohl es eigentlich unwahr ist, entgegen seiner eigenen »Theorie « nicht als Fälle von Lügen bezeichnen würde, können wir sagen, dass er den Ausdruck »lügen« versteht, obwohl er eine falsche Erklärung der Bedeutung angibt. Und würde man den Sprecher auf die entsprechenden Fälle hinweisen, wäre er sicher bereit, seine Theorie zu revidieren. Über eine Person, die zusätzlich zum Umstand, dass sie das Wort richtig verwendet, auch noch eine korrekte Definition von »lügen « angeben kann, könnte man unter Umständen dennoch sagen, dass sie den Ausdruck noch besser versteht als der oben angeführte Sprecher. Die Kompetenz im Umgang mit einem sprachlichen Ausdruck ist etwas Graduelles

71 Im Falle von Peter kommt noch eine seltsame Ansicht in Bezug auf die Existenz von Füchsinnen hinzu. 
und hängt unter anderem davon ab, (i) wie eine Person den Ausdruck im alltäglichen Leben gebraucht, (ii) wie sie auf Korrekturen ihres Gebrauchs reagiert, (iii) wie die Person die Bedeutung des Ausdrucks erklärt und (iv) wie sie auf Korrekturen ihrer Bedeutungserklärung reagiert. Wie weit eine Person auf der Skala der Kompetenz im Umgang mit einem Wort fortgeschritten sein muss, damit es korrekt ist, über sie zu sagen, dass sie den Ausdruck versteht, ist nicht genau festgelegt.

Zurück zu Peter und Stephen. Williamson macht also geltend, dass der Umstand, dass sie eine falsche Theorie darüber vertreten, wie der Ausdruck »alle« verwendet wird, damit kompatibel ist, dass sie den Ausdruck auf gewöhnliche Weise verwenden. So weit, so gut. Aber für Williamsons Argumentation ist es ja auch relevant, dass Peter und Stephen im Einklang mit ihrer falschen Theorie behaupten, das Urteil »Alle Füchsinnen sind Füchsinnen« sei unwahr. Und damit haben wir es bereits mit einer Abweichung in der Verwendung von »alle« zu tun, die über das bloße Formulieren einer falschen Theorie hinausgeht. Im Unterschied zum oben angeführten Sprecher, der das Wort »ügen« falsch definiert, halten Peter und Stephen außerdem auch dann an ihrer falschen Theorie fest, wenn sie mit Argumenten dagegen konfrontiert werden. ${ }^{72}$

Natürlich könnten wir uns auch einen Peter und einen Stephen vorstellen, die nur innerhalb von philosophischen Diskussionen behaupten, »Alle Füchsinnen sind Füchsinnen « sei unwahr und die Wahrheit von »Alle $F$ sind $G$ « setze die Existenz von mindestens einem $F$ resp. die Unmöglichkeit von Grenzfällen für das Fallen unter $» F \ll$ voraus, aber außerhalb philosophischer Diskussionen das Wort »alle« ganz normal und nicht im Einklang mit ihrer Theorie verwenden. Dann könnten wir über Peter und Stephen sagen, dass sie den Ausdruck »alle« im Alltag richtig verstehen, aber in philosophischen Diskussionen in Bezug auf die Bedeutung dieses Ausdrucks verwirrt sind. In dem Moment, in welchem sie den Satz »Alle Füchsinnen sind Füchsinnen« als unwahr deklarieren, würden sie ihn auch in diesem Szenario nicht richtig verstehen.

Zuletzt zu (h): Dass Peters und Stephens Verständnis von »Alle Füchsinnen sind Füchsinnen « semantisch vom gewöhnlichen Verständnis dieses Satzes abweicht, steht, wie bereits weiter oben erwähnt, schon deshalb außer Frage, weil sie dem Satz andere als die gewöhnlichen Wahrheitsbedingungen zuschreiben. Peter und Stephen zufolge ist das Urteil, das von »Alle Füchsinnen sind Füchsinnen« zum Ausdruck gebracht wird, teilweise empirisch und der Satz kann deshalb in ihrem Munde nicht bedeutungsgleich sein mit einem analytischen Urteil. Was logische Konstanten wie »alle«, »nicht« oder »und«

72 Vgl. Williamson 2007, S. 101. Vgl. zu diesem Punkt auch Nimtz 2009, S. 148 f. 
bedeuten, ist ebenso eine semantische Frage wie was Ausdrücke wie »Füchsin « oder »weiblich « bedeuten. Severin Schroeder versteht Williamson so, dass er die Abweichung von Peter und Stephen deshalb als logisch, und nicht als semantisch einordnet, weil die beiden Williamson zufolge einen Fehlschluss begehen. ${ }^{73} \mathrm{Im}$ Falle von Peter käme hierfür wohl der Fehlschluss von »Es gibt keine Füchsinnen « auf »Es ist nicht wahr, dass alle Füchsinnen Füchsinnen sind « in Frage. Aber wie Schroeder richtig bemerkt, sprechen wir normalerweise nur dann von einem Fehlschluss oder von einem deduktiven und mithin logischen Fehler, wenn der Umstand, dass jemand eine falsche Konklusion aus einer (Reihe von) Prämisse(n) gezogen hat, nicht darauf zurückführbar ist, dass diese Person eine idiosynkratische Auffassung von einer der Prämissen oder von der Konklusion hat. ${ }^{74}$ Peter und Stephen vertreten aber, wie Williamson selbst schreibt, eine abweichende Theorie über die Wahrheitsbedingungen von Allaussagen. Dass sie zur Konklusion gelangen, dass es nicht der Fall ist, dass alle Füchsinnen Füchsinnen sind, beruht also darauf, dass sie diese Konklusion anders interpretieren also wir, und nicht auf einem deduktiven Fehler, und ihre Abweichung ist somit nicht logischer, sondern semantischer Natur. ${ }^{75}$

Soviel zu Williamsons Argumenten dafür, dass Peter und Stephen den Satz »Alle Füchsinnen sind Füchsinnen « in seiner gewöhnlichen Bedeutung verstehen. In Amie L. Thomassons Besprechung der Fälle von Peter und Stephen findet sich als zusätzliches Argument gegen Williamsons Auffassung die Behauptung, die von Williamson aufgezählten Aspekte (a) bis (h) seien inkonsistent. Insbesondere die Aspekte (c) und (f) passen Thomasson zufolge nicht gut zueinander: (f) legt nahe, dass Peter und Stephen sich zu einem bestimmten Zeitpunkt auf der Grundlage von philosophischen Argumenten bewusst dafür entschieden haben, Allaussagen in Zukunft anders zu verwenden, als andere Leute (und bis zu diesem Zeitpunkt auch sie selbst) es mach $(t)$ en. Dieser Auffassung zufolge verfolgen Peter und Stephen eine sprachrevisionistische Absicht, und versuchen also - contra (c) - gerade nicht, die betreffenden Sätze auf gewöhnliche Art und Weise zu verwenden. ${ }^{76}$ Obschon ich diesen Punkt von Thomasson sehr interessant finde, bin ich nicht davon überzeugt, dass er stichhaltig ist. Den Punkt (c) formuliert Williamson nämlich nicht so, dass Peter und Stephen beabsichtigen, den Ausdruck »Alle Füchsinnen sind Füchsinnen« auf dieselbe Weise zu verwenden wie die anderen Leute.

73 Für diese Interpretation spricht zum Beispiel Williamson 2009, S. 102.

74 Vgl. Schroeder 2009, S. 88f.

75 Vgl. Schroeder 2009, S. 89. Vgl. für eine ähnliche Argumentation auch Glock 2010, S. 345.

76 Vgl. Thomasson 2015, S. 243 f. 
Sondern er sagt, sie beabsichtigen, ihn mit seiner gewöhnlichen Bedeutung $\mathrm{zu}$ verwenden und so wie es auch für die anderen Mitglieder ihrer Sprachgemeinschaft korrekt wäre. So ausbuchstabiert, ist (c) natürlich problemlos mit (f) vereinbar: Peter und Stephen können aufgrund von philosophischen Überlegungen zur Auffassung gelangt sein, dass sie und die anderen Mitglieder ihrer Sprachgemeinschaft den Ausdruck bisher - relativ zu den bereits geltenden Regeln - falsch verwendet haben und dass es für alle Sprecherinnen und Sprecher des Deutschen korrekt wäre, die Wahrheit von »Alle Füchsinnen sind Füchsinnen« zu bestreiten, wenn es keine Füchsinnen gibt bzw. wenn es Grenzfälle von Füchsinnen gibt oder geben kann. (Und selbst wenn man beweisen könnte, dass eine solche Auffassung einer kritischen Überprüfung nicht standhalten kann, würde dies nicht zeigen, dass Peter und Stephen die Auffassung nicht vertreten können.)

\subsubsection{Probleme epistemologischer Konzeptionen von Analytizität}

Aus den oben dargelegten Gründen ist Williamsons Argumentation dafür, dass es möglich ist, den Satz »Alle Füchsinnen sind Füchsinnen« zu verstehen, ohne ihm zuzustimmen, nicht überzeugend. Ich tendiere aber dennoch zur Ansicht, dass Williamson richtig damit liegt, dass das Nicht-Zustimmen zu einem analytischen Satz keine hinreichende Bedingung dafür ist, den Satz nicht oder falsch zu verstehen. Stellen wir uns einen Philosophie-Studenten im ersten Semester vor, der in der Logik-Vorlesung etwas falsch verstanden hat und glaubt, man habe ihm erklärt, dass Sätze der Form »Alle $F$ sind $F$ « unwahr sind, wenn es keine $F$ s gibt. Der Student stimmt deshalb dem Satz »Alle Zentauren sind Zentauren« nicht mehr zu, hat aber auf die Frage, weshalb dieser Satz unwahr sein sollte, keine befriedigende Antwort. Er sagt, er könne sich an den Grund dafür nicht mehr erinnern. Weiter können wir uns vorstellen, dass dieser Student die Bedeutung des Satzes immer noch gleich erklären würde wie vor dem Besuch der Vorlesung und nach wie vor den Impuls hätte, Sätzen der Form »Alle $F$ sind $F \ll$ auch dann zuzustimmen, wenn es keine $F$ s gibt - bloß würde er diesen Impuls neuerdings unterdrücken. Auf der Skala zwischen Überhaupt-nicht-Verstehen und Perfekt-Verstehen könnte dieser Student nicht mehr an der Spitze des Verstehens angesiedelt werden. Er könnte nämlich der Behauptung, der Satz sei genau dann wahr, wenn es keinen Zentauren gibt, der kein Zentaur ist, nicht mehr vorbehaltlos zustimmen. Dies stünde in einem zu offensichtlichen Widerstreit zur Konjunktion seiner Überzeugungen, dass es (a) keinen Zentauren gibt, der kein Zentaur ist, und (b) dass der Satz »Alle Zentauren sind Zentauren« unwahr ist. Alles in allem wäre ich aber dennoch dazu bereit, über diesen Studenten zu sagen, dass er den Satz »Alle Zentauren sind Zentauren« hinreichend gut versteht, um als Beispiel für 
eine Person zu gelten, die einen bestimmten analytischen Satz versteht, ohne ihm zuzustimmen.

In einer sehr wohlwollenden Rezension von The Philosophy of Philosophy geht die amerikanische Philosophin Gillian Russell noch einen Schritt weiter und schreibt, es sei von Beginn an unerklärlich, wie sich in der Philosophie die Idee verbreiten konnte, die Analytizität von Urteilen an ein psychologisches Phänomen, nämlich Zustimmung, zu knüpfen. Weitaus aussichtsreicher wäre es, so Russell, zur Definition von Analytizität den epistemischen Begriff der Rechtfertigung heranzuziehen. ${ }^{77} \mathrm{Um}$ zu zeigen, dass es keinen zwingenden Zusammenhang zwischen Verstehen und Zustimmung gibt, benötigen wir Russell zufolge gar keine komplexen Fälle wie die von Peter und Stephen (die Russell allerdings als einschlägig betrachtet), sondern können ganz simple Beispiele hervorbringen:

[I]f Sally understands "all vixens are vixens," but I threaten to shoot her if she assents to it, she may have herself hypnotized into refraining from assenting to it. Posthypnosis Sally is a counterexample to $\left(\mathrm{UAl}^{\prime 78}\right)$ as well. So much the worse for $\left(\mathrm{UAl}^{\prime}\right) .{ }^{79}$

Russell irrt sich allerdings darin, dass es sich bei der Zustimmung zu analytischen Urteilen einfach um ein psychologisches Phänomen handelt, das von semantischen und epistemischen Belangen unabhängig ist. Tatsächlich ist die Zustimmung zu einem analytischen Urteil ein Kriterium (im wittgensteinianischen Sinn) dafür, dass die zustimmende Person das Urteil versteht: ${ }^{80}$ Die Regeln für die Verwendung von »verstehen « legen fest, dass es für die Beurteilung davon, ob jemand einen analytischen Satz versteht oder nicht, eine Rolle spielt, ob diese Person dem Satz zustimmt oder nicht. Es liegt in der Bedeutung von »verstehen, was >Alle Füchsinnen sind Füchsinnen< bedeutet«, dass die Nicht-Zustimmung zum Satz »Alle Füchsinnen sind Füchsinnen« ein Grund dafür ist, daran zu zweifeln, dass die betreffende Person diesen Satz versteht. Und dieser begriffliche Zusammenhang zwischen der Zustimmung zu einem analytischen Satz und dem Verstehen desselben verbindet den Begriff der Zustimmung mit einer epistemischen Kategorie. Denn das Verstehen

\footnotetext{
77 Vgl. Russell (2010), S. 44.

78 Mit »UAl'« bezieht sich Russell auf das Urteil »Es ist notwendig, dass jeder, der den Satz >All vixens are vixens` versteht, ihm zustimmt.«

79 Russell 2010, S. 44.

8o Vgl. Glock 1996a, Eintrag »criteria«. Im Übrigen ergibt sich die konventionelle Bedeutung sprachlicher Ausdrücke auch unter anderem daraus, welchen Sätzen die Mitglieder einer Sprachgemeinschaft zustimmen. Aber das ist für den vorliegenden Kontext weniger wichtig.
} 
eines sprachlichen Ausdrucks ist dasselbe wie das Wissen, was der Ausdruck bedeutet. Das zur Diskussion stehende Prinzip »Ein Satz ist genau dann analytisch, wenn es nicht möglich ist, ihn zu verstehen, ohne ihm zuzustimmen « ist also nicht so abwegig wie Russell glaubt.

Aufgrund des begrifflichen Zusammenhangs zwischen Zustimmung und Verstehen können wir entgegen dem, was Russell mit ihrem Hypnose-Beispiel nahelegt, auch nicht einfach an jemandes Zustimmung zu einem Urteil schrauben und dabei voraussetzen, dass das sprachliche Verstehen dieser Person davon unberührt bleibt. Russell schuldet uns eine Reihe von zusätzlichen Informationen über den von ihr entworfenen Fall, die für die Frage relevant sind, ob Sally nach der Hypnose tatsächlich immer noch versteht, was der Satz »Alle Füchsinnen sind Füchsinnen« bedeutet. Wie würde Sally die Bedeutung dieses Satzes erklären? Wie würde sie begründen, dass der Satz unwahr ist? Es ist nicht selbstverständlich, dass das Sally-Beispiel so ausbuchstabiert werden kann, dass wir mit Sally ein konsistentes Beispiel einer Person haben, die »Alle Füchsinnen sind Füchsinnen« versteht, aber diesem Satz nicht zustimmt.

$4 \cdot 3 \cdot 3$

Annullierbare Kriterien und die Abgrenzung analytischer Sätze zu Angelsätzen

Die Zustimmung zu einem analytischen Satz ist, wie oben erwähnt, ein Kriterium für das Verstehen dieses Satzes, aber keine notwendige Bedingung dafür. Hacker schreibt, bezugnehmend auf Grice und Strawson, völlig richtig, das Nicht-Zustimmen zu einem analytischen Urteil sei ein annullierbares, logisches Kriterium (»a defeasible logical criterion«) für das Nicht-Verstehen des Urteils. ${ }^{81}$ Das heißt, es ist möglich, ein analytisches Urteil zu verstehen, ohne ihm zuzustimmen, aber wenn wir einen zur Diskussion stehenden Fall auf diese Weise charakterisieren wollen, dann müssen wir eine Geschichte dazu erzählen können, wie es zu einem solchen Sachverhalt kommen konnte. Denn im Normalfall stimmt eine Person, die ein analytisches Urteil versteht, diesem Urteil auch zu. Da das Kriterium der Nicht-Zustimmung aber eben annullierbar ist, stimmt es nicht, dass ein Urteil genau dann analytisch ist, wenn man es nicht verstehen kann, ohne ihm zuzustimmen. Wenn wir zusätzlich zur Definition analytischer Urteile als solche Urteile, die allein aufgrund ihrer Bedeutung wahr sind, auch noch eine epistemologische Definition analytischer Wahrheiten haben wollen, müssen wir uns also etwas anderes überlegen.

$81 \quad$ Vgl. Hacker 2009a, S. 346. 
Ein möglicher Ansatzpunkt bestünde darin, auf die im vorangehenden Absatz dargelegte Erkenntnis zurückzugreifen und analytische Sätze wie folgt zu definieren:

Ein Satz ist genau dann analytisch, wenn die Nicht-Zustimmung zu dem Satz ein annullierbares, logisches Kriterium für das Nicht-Verstehen desselben ist.

Das Problem mit dieser Definition ist, dass ihr zufolge auch so genannte »Angelsätze $«^{82}$ (hinge propositions) oder überhaupt alle offensichtlich wahren Sätze als analytisch klassifiziert würden. Denn auch dass jemand den folgenden Sätzen nicht zustimmt, ist ein annullierbares, logisches Kriterium dafür, dass diese Person die Sätze nicht versteht:

(12) Das Universum hat gestern schon existiert.

(13) Es gibt Menschen.

(14) Feuer ist heiß.

Aber die Sätze (12), (13) und (14) sind nicht analytisch. Ich würde deshalb dafür plädieren, bei einer semantischen Definition der Analytizität zu bleiben und analytische Sätze als solche Sätze zu definieren, die wahr sind aufgrund ihrer Bedeutung.

Ein weiterer Aspekt, der dafür spricht, Analytizität semantisch statt epistemologisch zu definieren, ergibt sich durch die bereits in früheren Kapiteln angesprochene Existenz synoptischer begrifflicher Wahrheiten. Während es bei trivialen begrifflichen Wahrheiten wie »Alle Füchsinnen sind Füchsinnen« oder »Ein Enterich ist eine männliche Ente« einleuchtend ist, dass die Weigerung, ihnen zuzustimmen, dafür spricht, dass man die Urteile nicht richtig versteht, ist dies bei »Es kann keine private Sprache geben « oder »Ein Wesen ohne eine Sprache kann keine Überzeugungen darüber haben, was übermorgen passiert « weniger plausibel: ${ }^{83}$ Muss ich, um zu verstehen, was »Es kann keine private Sprache geben « bedeutet, wirklich einen Überblick haben über die Zusammenhänge zwischen den Begriffen der Sprache, der Bedeutung, der Regel, usw.? Man könnte zumindest dafür argumentieren, dass dieser Anspruch überzogen wäre. Aber selbst wenn man behaupten wollte, dass man Sätze wie »Es kann keine private Sprache geben« oder »Ein Wesen ohne eine Sprache kann keine Überzeugungen darüber haben, was übermorgen passiert « nicht vollständig versteht, wenn man nicht einsieht, dass (und weshalb) sie

82 Angelsätze bilden die Angeln, um die sich unsere Sprachspiele drehen ( $\ddot{U} G$ §§ 341-343). Sie sind synthetische Sätze, deren Wahrheit sich nicht beweisen lässt, da jede Evidenz, die für sie angeführt werden könnte, weniger gewiss ist als die Wahrheit der Angelsätze selbst. Vgl. Glock 2016, insb. Abschnitt 4.

83 Vgl. dazu auch Schroeder 2009, S. 85 . 
wahr sind, könnte ein epistemologisches Kriterium der oben dargelegten Art doch nicht im gleichen Maße operativ sein wie ein semantisches Kriterium: Um herauszufinden, ob der Satz »Es kann keine private Sprache geben« das epistemologische Kriterium erfüllt und also zu der Sorte von Sätzen gehört, im Falle derer Nicht-Zustimmung ein Kriterium für Nicht-Verstehen ist, müssen wir prüfen, ob der Satz wahr aufgrund seiner Bedeutung ist. Das Kriterium, das wir bei synoptischen begrifflichen Wahrheiten eigentlich zur Klassifikation verwenden, ist also in jedem Fall das semantische.

\subsection{Inkonsistente Überzeugungen?}

Wie im vorangehenden Abschnitt dargelegt, argumentiert Williamson dafür, dass es möglich ist, einen analytischen Satz wie »Alle Füchsinnen sind Füchsinnen « zu verstehen und gleichzeitig zu glauben, dass dieser Satz unwahr ist. Ein bisher noch nicht angesprochenes Problem dieser Auffassung ergibt sich im Zusammenhang mit der Bestimmung davon, was eine Person genau glaubt, wenn sie glaubt, dass »Alle Füchsinnen sind Füchsinnen« unwahr ist. Über den Studenten, den ich zu Beginn des Abschnitts 4.3.2 als Beispiel für jemanden angeführt habe, der den Satz »Alle Füchsinnen sind Füchsinnen « versteht, ohne dem Satz zuzustimmen, würde ich sagen, dass er ein metasprachliches Urteil glaubt: Er glaubt, dass der Satz »Alle Füchsinnen sind Füchsinnen« unwahr ist. Zu sagen, er glaube, dass nicht alle Füchsinnen Füchsinnen sind, wäre Unsinn, da »Nicht alle Füchsinnen sind Füchsinnen« Unsinn ist. Man kann zwar glauben, dass »Nicht alle Füchsinnen sind Füchsinnen« wahr ist, aber man kann nicht glauben, dass nicht alle Füchsinnen Füchsinnen sind: »Nicht alle Füchsinnen sind Füchsinnen« vermag überhaupt keinen Überzeugungsinhalt zu bestimmen. ${ }^{84}$

Williamson wendet sich allerdings explizit gegen eine solche metasprachliche Interpretation davon, was die Protagonisten seiner Gedankenexperimente glauben. Über Peter und Stephen schreibt er: »They genuinely doubt that every vixen is a vixen $\ll^{85} \mathrm{und}$ :

Since a homophonic reading of (1) [i. e. 'Every vixen is a vixen'] in the mouths of Peter and Stephen is more faithful to their intentions than is any non-homophonic reading, they associate (1) with the same thought as we do in any relevant sense of "thought." 86

84 NB: Ich möchte nicht prinzipiell in Abrede stellen, dass es möglich ist, inkonsistente Überzeugungen zu haben. Aber es ist meines Erachtens nur möglich im Falle von Überzeugungen, bei denen der Beweis ihrer Inkonsistenz eine komplexere Angelegenheit ist als bei »Nicht alle Füchsinnen sind Füchsinnen«.

85 Williamson 2007 , S. 91.

86 Williamson 2007, S. 115 . 
Weiter unten im selben Kapitel von The Philosophy of Philosophy, in einer Passage, in welcher es um eine Person geht, die glaubt, dass »Alle unverheirateten Männer sind Junggesellen « unwahr ist, ${ }^{87}$ buchstabiert er seine Gründe dagegen, die betreffende Überzeugung metasprachlich zu interpretieren, im Detail aus: Im betreffenden Fall geht es um eine Person, die glaubt, ein Mann, der seit Jahren mit einer Partnerin oder einem Partner zusammenlebe, ohne eine Ehe einzugehen, zähle nicht als Junggeselle. Williamson macht geltend, dass wir die Überzeugung dieser Person mit den Worten »Sie glaubt, dass einige unverheiratete Männer keine Junggesellen sind « beschreiben könnten. Dass wir in dieser Beschreibung die Wörter »unverheiratet« und »Junggeselle « verwenden würden (ohne sie in Anführungszeichen zu setzen), zeige, dass wir davon ausgehen, dass die fragliche Person diese Wörter in ihrem gewöhnlichen deutschen Sinn auffasst. ${ }^{88}$ Und weiter unten führt er aus:

Arguably their error is not primarily semantic: they have the semantic belief that the word "bachelor" does not apply to all unmarried men because they have the non-semantic belief that some unmarried men are not bachelors and the semantic knowledge that "bachelor" applies only to bachelors[.] ${ }^{89}$

Tatsächlich ergibt »Sie glaubt, dass einige unverheiratete Männer keine Junggesellen sind « aber nur dann Sinn, wenn wir die Überzeugung (unter anderem) metasprachlich interpretieren, nämlich im Sinn von »Sie glaubt, dass der Ausdruck >Junggeselleく nicht auf alle unverheirateten Männer zutrifft.« Was die von Williamson im obigen Zitat erwähnte »nicht-semantische Überzeugung«, dass einige unverheiratete Männer keine Junggesellen sind, sein sollte, ist vollkommen unverständlich. Natürlich können wir uns im zur Diskussion stehenden Kontext nicht-semantische Überzeugungen vorstellen. Zum Beispiel die Überzeugung, dass man bestimmte unverheiratete Männer, nämlich beispielsweise eben Männer, die seit Jahren verpartnert sind, nicht als Junggesellen bezeichnen sollte, weil dies am Witz des Begriffs Junggeselle vorbeigeht, oder die Überzeugung, dass einige unverheiratete Männer nicht auf dem Partnermarkt zur Verfügung stehen. Aber weder die eine noch die andere Überzeugung könnte man mit den Worten »Einige unverheiratete Männer sind keine Junggesellen « wiedergeben.

Williamson verweist in seiner Argumentation dagegen, die Behauptung »Nicht alle unverheirateten Männer sind Junggesellen« metasprachlich zu

87 Dieser Satz ist tatsächlich unwahr, da beispielsweise Pfarrer unverheiratet sein können, ohne unter den Begriff des Junggesellen zu fallen. Diese Komplikation werde ich allerdings nachfolgend ignorieren.

88

Vgl. Williamson 2007, S. 118 .

89 Williamson 2007 , S. 119 . 
interpretieren, unter anderem auf Tyler Burges Artikel »Belief and Synonymy« (1978), ohne einen Hinweis darauf zu geben, wie Burge in diesem Artikel argumentiert. Dies soll hier nachgeholt werden. Bei Burge findet sich zunächst einmal ein Argument, das als Einwand gegen meine obige Behauptung, es sei nicht möglich, dass Peter und Stephen glauben, dass nicht alle Füchsinnen Füchsinnen sind (aber wohl möglich, dass sie glauben, dass »Alle Füchsinnen sind Füchsinnen« unwahr ist), angeführt werden könnte. Auf dieses Beispiel übertragen, funktioniert Burges Argument wie folgt: ${ }^{90}$ Gehen wir davon aus, dass wir Peter und Stephen zugestehen, das folgende Urteil zu glauben:

(15) »Alle Füchsinnen sind Füchsinnen« ist unwahr.

Solange wir Peter und Stephen außerdem wenigstens als minimal sprachkompetent einstufen, müssen wir annehmen, dass sie auch dem folgenden Urteil zustimmen:

(16) »Alle Füchsinnen sind Füchsinnen« ist genau dann unwahr, wenn nicht alle Füchsinnen Füchsinnen sind.

Aus (15) und (16) folgt allerdings deduktiv (17):

(17) Nicht alle Füchsinnen sind Füchsinnen.

Also sollte es Peter und Stephen doch auch möglich sein, (17) zu glauben. Und analog für die Person, die glaubt, dass Männer, die zwar unverheiratet sind, aber sich in langjährigen Beziehungen befinden, keine Junggesellen sind. Diese Person glaubt offenbar (18):

(18) Nicht alle unverheirateten Männer fallen unter den Ausdruck »Junggeselle «.

Und als sprachkompetente Person wird sie auch dem folgenden Urteil zustimmen:

(19) Ein Ding (bzw. eine Person) fällt genau dann unter den Ausdruck »Junggeselle«, wenn es (bzw. sie) ein Junggeselle ist.

(18) und (19) implizieren aber wiederum (20):

(20) Nicht alle unverheirateten Männer sind Junggesellen.

Wenn es einer Person möglich ist, (18) und (19) zu glauben, sollte diese Person also auch (20) glauben können. Da (20) im Gegensatz zu (17) nicht inkonsistent ist, ${ }^{91}$ gibt Burges Konklusion in diesem Fall nicht zu Problemen Anlass, deshalb sollten wir für die weitere Besprechung lieber auf ein anderes Beispiel zurückgreifen:

(21) Nicht alle weiblichen Füchse fallen unter den Ausdruck »Füchsin«.

(22) Ein Ding fällt genau dann unter den Ausdruck»Füchsin«, wenn es eine Füchsin ist.

9o Vgl. Burge 1978, S. 123.

$91 \quad$ Vgl. Fußnote 87. 
(23) Nicht alle weiblichen Füchse sind Füchsinnen.

Die Idee wäre dann auch hier, dass es problematisch ist, zu behaupten, dass es zwar möglich sei, (21) zu glauben, aber nicht, (23) zu glauben, da (23) aus der Konjunktion von (21) mit dem unkontroversen Urteil (22) folgt.

Abgesehen davon, dass es, wie bereits erwähnt, gar nicht klar ist, was es bedeuten würde, (den Inhalt von) (17) oder (23) zu glauben (zumindest dann nicht, wenn wir für (23) eine metasprachliche Interpretation ausschließen), scheitert die Stichhaltigkeit von Burges Argumenten jeweils am zweiten Schritt, also an seinen Ausführungen zu den Urteilen (16) und (22): Zwar stimmt es, dass jede minimal sprachkompetente Person diesen beiden Sätzen zustimmen würde oder überhaupt allen Sätzen der Form

(24) $» \mathrm{p} \ll$ ist genau dann wahr, wenn $p$. bzw.

(25) Ein Ding fällt genau dann unter den Ausdruck $»$ F «, wenn es $F$ ist. Aber das heißt nicht, dass sprachkompetente Personen auch allen diesen Urteilen mit Verständnis zustimmen. Denn ob eine Person einen einzelnen Satz der Form (24) oder (25) versteht, hängt unter anderem davon ab, ob diese Person den Ausdruck versteht, der für »p« bzw. »F« eingesetzt wird. Eine Person, die das Wort »alle« falsch versteht, versteht auch den Satz (16) falsch. Und eine Person, die das Wort »Füchsin « falsch versteht, versteht auch den Satz (22) falsch. Solche Personen würden über (16) und (22) also zwar sagen, dass die beiden Sätze wahr sind, aber sie würden die Sätze eben nicht richtig verstehen, und wir wären deshalb nicht dazu gezwungen, zu sagen, dass (16) und (22) Überzeugungen solcher Personen zum Ausdruck bringen und dass ihre gesammelten Überzeugungen somit die inkonsistenten Aussagen (17) oder (23) implizieren. Und dass eine Person den Sätzen (15) beziehungsweise (21) zustimmt, zeigt bereits, dass sie nicht über vollständige Kenntnis der Bedeutung der relevanten Ausdrücke verfügt. Also können wir überhaupt keine Person konsistent beschreiben, die sowohl den Satz (15) als auch den Satz (16) respektive sowohl den Satz (21) als auch den Satz (22) vollständig versteht und ihnen beiden zustimmt. Und somit gelingt es Burge nicht, zu beweisen, dass es möglich ist, die Urteile (17) und (23) zu glauben. ${ }^{92}$

92 Es ist nicht ganz klar, ob das Argumentationsziel von Burge für Williamsons Zwecke überhaupt weitreichend genug ist. Obwohl Burge sich dafür ausspricht, Sprecherinnen und Sprecher, die Sätzen wie »Einige weibliche Füchse sind keine Füchsinnen « beim Wort zu nehmen bzw. ihre Behauptungen »at face value« zu nehmen (vgl. Burge 1978, S.126, 134) und sie nicht metasprachlich »umzuinterpretieren «, räumt er explizit ein, die betreffenden Überzeugungen dieser Sprecherinnen und Sprecher handelten in einem gewissen Sinn von sprachlichen Ausdrücken und seien auf sprachliche Irrtümer zurückzuführen (vgl. Burge 1978, S. 128f.). 
Burge führt aber auch vom obigen Argument unabhängige Gründe gegen die Idee an, dass (23) als Spezifikation einer Überzeugung in jedem Fall metasprachlich interpretiert werden muss. Er macht beispielsweise geltend, dass eine Person, die ein Urteil wie (23) glaubt, überhaupt keine metasprachlichen Überzeugungen haben muss: Um der Überzeugung zu sein, dass einige weibliche Füchse keine Füchsinnen sind, so Burge, werden keine semantischen Begriffe wie etwa der Begriff der Bedeutung benötigt. ${ }^{93}$ Damit spricht Burge einen interessanten Punkt an. Tatsächlich können wir uns zum Beispiel ein Kind vorstellen, das noch nicht gelernt hat, die Ausdrücke »Bedeutung «, »sich beziehen auf «, »Sprache« usw. zu verwenden, aber dem trotzdem bereits die Überzeugung zugeschrieben werden kann, dass nicht alle weiblichen Füchse Füchsinnen sind. Wir können die Überzeugung diesem Kind zum Beispiel auf der Grundlage zuschreiben, dass es beide Ausdrücke, »weiblicher Fuchs« und »Füchsin«, manchmal auf Füchsinnen anwendet, aber wenn wir es bei bestimmten Tieren, die es als weibliche Füchse bezeichnet hat, fragen, ob diese Tiere auch Füchsinnen seien, diese Frage verneint. Dies zeigt jedoch nur, dass es möglich ist, semantische Überzeugungen zu haben, ohne den Begriff der Bedeutung zu besitzen. Denn die Bereitschaft, bestimmte Dinge, aber nicht andere als »Füchsin« zu bezeichnen, manifestiert eine bestimmte Auffassung der Bedeutung dieses Wortes. Auch Personen, die keine explizit metasprachlichen Überzeugungen formulieren können, können also solche Überzeugungen besitzen.

Die restliche Argumentation von Burge funktioniert so, dass er behauptet, dass wir - entgegen einem Eindruck, der bisweilen in Texten der analytischen Philosophie erweckt werde - wenigstens ein Argument dafür brauchen, Äußerungen von Sätzen wie (23) metasprachlich zu interpretieren, statt sie wörtlich zu nehmen. Er versucht dann, zu zeigen, dass keines der Argumente, die bisher für eine solche Uminterpretation angeführt oder angedeutet wurden, stichhaltig sind. ${ }^{94}$ Das interessanteste Argument zugunsten einer metasprachlichen Interpretation, mit dem sich Burge auseinandersetzt, ergibt sich aus Quines principle of charity: Wenn jemand etwas vollkommen Irrationales zu behaupten scheint, sollten wir versuchen, seine Worte anders zu interpretieren, so dass sich eine vernünftigere Behauptung ergibt. Da es extrem irrational wäre, ein inkonsistentes Urteil wie »Einige weibliche Füchse sind keine Füchsinnen « zu glauben, sollten wir lieber davon ausgehen, dass eine Person, die diesen Satz äußert, eigentlich etwas Metasprachliches

93 Vgl. Burge 1978, S. 128, Fußnote 10.

94 Vgl. Burge 1978, S. 126-132. 
ausdrücken will, das zwar falsch, aber nicht inkonsistent ist. ${ }^{95}$ Diesem Argument hält Burge entgegen, dass es für Personen, die eine falsche Auffassung der Bedeutung von »Füchsin« haben, überhaupt nicht irrational ist, zu glauben, dass einige weibliche Füchse keine Füchsinnen sind, auch wenn diese Überzeugung inkonsistent ist. ${ }^{96}$ Das principle of charity ist also Burge zufolge ohne Weiteres vereinbar damit, solchen Leuten die nicht metasprachlich uminterpretierte Überzeugung (23) zuzuschreiben.

Auch hier und überhaupt im Kontext mit der gesamten Argumentationslinie von Burge ergibt sich wieder das Problem, dass überhaupt nicht klar ist, was es bedeuten würde, (23) als jemandes Überzeugungsinhalt »wörtlich« zu nehmen, da der Satz, nicht metasprachlich interpretiert, keinen Sinn ergibt. Und dies scheint mir bereits ein zwingendes Argument für eine metasprachliche »Uminterpretation « zu sein. Wenn wir die Überzeugung auf einen sprachlichen Irrtum zurückführen können und, wie Burge weiter unten schreibt, durch die Konsultation eines Wörterbuchs widerlegen können, ${ }^{97}$ dann erscheint es mir vollkommen offensichtlich, dass wir es mit einer konsistenten, metasprachlichen Überzeugung zu tun haben. Auch bei Burge finden sich also keine stichhaltigen Argumente zur Rettung von Williamsons Position.

\section{5} Schluss

Im vorliegenden Kapitel habe ich mich hauptsächlich mit den folgenden drei Thesen von Williamson auseinandergesetzt:

1. Philosophische Probleme handeln in der Regel weder explizit noch implizit von sprachlichen Ausdrücken oder Begriffen.

2. Es gibt keine Sätze, die allein aufgrund ihrer Bedeutung wahr sind.

3. Analytizität lässt sich nicht adäquaterweise epistemologisch definieren, da es sogar bei logischen Wahrheiten wie »Alle Füchsinnen sind Füchsinnen « möglich ist, sie zu verstehen, ohne ihnen zuzustimmen.

In Bezug auf (1) habe ich geltend gemacht, dass das wichtigste Argument dafür, über eine Frage wie »Ist jeder Gegenstand zu jedem Zeitpunkt entweder trocken oder nicht trocken? « zu sagen, dass sie von Wörtern oder Begriffen handelt, darin besteht, dass wir uns zur Beantwortung dieser Frage nicht mit der Untersuchung von Gegenständen in der Welt befassen müssen,

95 Vgl. Burge 1978, S. $128 f$.

96 Burge bezieht sich dabei auf ein anderes Beispiel, aber das spielt für die Argumentation keine Rolle.

97 Vgl. Burge 1978, S. 130. 
sondern damit, wie die Ausdrücke »trocken« und »nicht trocken« korrekterweise verwendet werden. Williamson versucht, zu zeigen, dass wir uns auch zur Beantwortung von Fragen, die eindeutig von der Welt handeln, manchmal nur oder hauptsächlich mit sprachlichen Ausdrücken befassen müssen, aber zwischen den Beispielen, die er in diesem Kontext anführt, und dem oben genannten philosophischen Beispiel gibt es entscheidende Disanalogien.

Im Zusammenhang mit (2) habe ich verschiedene Interpretationen von »wahr aufgrund von Bedeutung « unterschieden und besprochen. Selbstverständlich hat Williamson Recht damit, dass beispielsweise der Satz »Alle Füchsinnen sind weiblich « nicht deshalb wahr ist, weil er bedeutet: Alle Füchsinnen ist weiblich. Es ist aber nicht unplausibel, zu sagen, dass er wahr ist aufgrund der Bedeutung der einzelnen, in ihm vorkommenden Wörtern und der Art und Weise ihrer Zusammenfügung. Es gibt außerdem Lesarten der berühmten Behauptung »Synthetische Wahrheiten sind wahr aufgrund (a) ihrer Bedeutung und (b) von Tatsachen, analytische Wahrheiten sind wahr aufgrund ihrer Bedeutung allein«, nach welchen diese Aussage relativ unproblematisch ist und contra Williamson keine Äquivokation involviert, auch wenn ich dafür argumentiert habe, stattdessen zu sagen: Analytische Wahrheiten sind wahr aufgrund ihrer Bedeutung, synthetische Wahrheiten sind wahr aufgrund von Tatsachen.

In Bezug auf (3.) habe ich schließlich versucht, zu zeigen, dass Williamsons Peter und Stephen sich nicht als Beispiele für Personen eignen, die den Satz »Alle Füchsinnen sind Füchsinnen« verstehen, ohne ihm zuzustimmen. Außerdem habe ich geltend gemacht, dass Williamsons diesbezügliche Argumentation ebenso wie Tyler Burges Argumentation in einem Artikel, auf den sich Williamson beruft, bereits daran scheitert, dass überhaupt nicht klar ist, was es bedeuten würde, zu glauben, dass nicht alle Füchsinnen Füchsinnen sind. 